\title{
Plant data visualization using network graphs
}

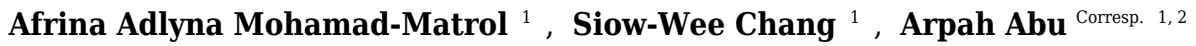 \\ 1 Institute of Biological Sciences, Faculty of Science, University of Malaya, Kuala Lumpur, Malaysia \\ Kuala Lumpur, Malaysia \\ Corresponding Author: Arpah Abu \\ Email address: arpah@um.edu.my
}

Background. The amount of plant data such as taxonomical classification, morphological characteristics, ecological attributes and geological distribution in textual and image forms has increased rapidly due to emerging research and technologies. Therefore, it is crucial for experts as well as the public to discern meaningful relationships from this vast amount of data using appropriate methods. The data are often presented in lengthy texts and tables, which make gaining new insights difficult. The study proposes a visual-based representation to display data to users in a meaningful way. This method emphasises the relationships between different data sets.

Method. This study involves four main steps which translate text-based results from XML serialization format into graphs. The four steps include: (1) conversion of ontological dataset as graph model data; (2) query from graph model data; (3) transformation of text-based results in XML serialization format into a graphical form; and (4) display of results to the user via a graphical user interface (GUI). Ontological data for plants and samples of trees and shrubs were used as the dataset to demonstrate how plant-based data could be integrated into the proposed data visualisation.

Results. A visualisation system named PlantViz was developed. This system provides a graphical user interface that enables users to perform the query process, as well as a graphical viewer to display the results of the query in the form of a network graph. The efficiency of the developed visualisation system was measured by performing two types of user evaluations: a usability heuristics evaluation, and a query and visualisation evaluation.

Discussion. The relationships between the data were visualised, enabling the users to easily infer the knowledge and correlations between data. The results from the user evaluation show that the proposed visualisation system is suitable for both expert and novice users, with or without computer skills. This technique demonstrates the practicability of using a computer assisted-tool by providing cognitive analysis for understanding relationships between data. Therefore, the results benefit not only botanists, but also novice users, especially those that are interested to know more about plants. 


\section{Plant Data Visualisation using Network Graphs}

Afrina Adlyna Mohamad-Matrol ${ }^{1}$, Siow-Wee Chang ${ }^{1}$, Arpah $\mathrm{Abu}^{1,2}$

${ }^{1}$ Institute of Biological Sciences, Faculty of Science, University of Malaya, Kuala Lumpur, Malaysia

${ }^{2}$ Centre of Research for Computational Sciences and Informatics for Biology, Bioindustry, Environment, Agriculture and Healthcare, University of Malaya, Kuala Lumpur, Malaysia

Corresponding Author:

Arpah $\mathrm{Abu}^{1,2}$

Institute of Biological Sciences, Faculty of Science, University of Malaya, Kuala Lumpur, 50603, Malaysia Email address: arpah@um.edu.my 
1 Abstract

2

3 Background. The amount of plant data such as taxonomical classification, morphological

4 characteristics, ecological attributes and geological distribution in textual and image forms has

5 increased rapidly due to emerging research and technologies. Therefore, it is crucial for

6 experts as well as the public to discern meaningful relationships from this vast amount of data

7 using appropriate methods. The data are often presented in lengthy texts and tables, which

8 make gaining new insights difficult. The study proposes a visual-based representation to

9 display data to users in a meaningful way. This method emphasises the relationships between

10 different data sets.

11 Method. This study involves four main steps which translate text-based results from XML

12 serialization format into graphs. The four steps include: (1) conversion of ontological dataset

13 as graph model data; (2) query from graph model data; (3) transformation of text-based results

14 in XML serialization format into a graphical form; and (4) display of results to the user via a 15 graphical user interface (GUI). Ontological data for plants and samples of trees and shrubs

16 were used as the dataset to demonstrate how plant-based data could be integrated into the 17 proposed data visualisation.

18 Results. A visualisation system named PlantViz was developed. This system provides a 19 graphical user interface that enables users to perform the query process, as well as a graphical 20 viewer to display the results of the query in the form of a network graph. The efficiency of the 21 developed visualisation system was measured by performing two types of user evaluations: a 22 usability heuristics evaluation, and a query and visualisation evaluation.

23 Discussion. The relationships between the data were visualised, enabling the users to easily 24 infer the knowledge and correlations between data. The results from the user evaluation show 
25 that the proposed visualisation system is suitable for both expert and novice users, with or

26 without computer skills. This technique demonstrates the practicability of using a computer

27 assisted-tool by providing cognitive analysis for understanding relationships between data.

28 Therefore, the results benefit not only botanists, but also novice users, especially those that are

29 interested to know more about plants.

\section{Introduction}

33 Plants play an important role and benefit all forms of life. In total, 452 vascular plant families

34 and $\sim 381,910$ species have been identified by botanists across the world, as reported by Willis

35 (2017). The biological field is full of diversified data inputs (Marx, 2013). Plant data range

36 from complete genome sequences to geographical information of plant species distribution

37 (Armstead et al. 2009; Hughes 2006). In addition, advancement in research technology and

38 methodology has caused plant data to increase rapidly. However, shortage of skill and time to

39 analyse these data points continue to be significant obstacles. Relationships between data are

40 often left out, which eventually produce less valuable information. In addition, any constraints

41 in textually inspecting the massive amount of data collected may cause valuable points to be

42 discarded (Keim 2002). Associating data that is related to one another however will point out

43 unique points of the data, which will provide new information on various knowledge domains.

44 Hence, it is important for researchers to interpret valuable data and present it in an engaging

45 format so that it is easy for the community to understand. This is because viewing data in

46 plain form such as in texts and tables will not be sufficient to provide clear explanation.

Data visualisation is a comprehensive field involving the crossover between 
49 2014). Data visualisation is defined as the representation of data using a visual or artistic

50 approach rather than the traditional reporting method (Yuk \& Diamond 2014). Data

51 visualisation plays an important role in many fields such as business (Tegarden 1999),

52 geography (Groenendyk 2013) and biology (Chen et al. 2014; Jensen \& Papin 2014; Sedova et

53 al. 2016). Visualised data better conveys the unique properties of the data it represents. In

54 particular, visualising biological data helps researchers to view the data from a different angle,

55 leading to new insights. This is because data shown via graphical representation makes it

56 easier for humans to conduct data analysis, as it provides more cognitive support (Tory \&

57 Moller 2004).

58 The key to effective data visualisation is the selection of the right type of visualisation

59 to match the type of data used such as charts, network graphs, Sankey diagrams, and tree

60 maps. Most data visualisations are interactive, enabling users to manipulate and explore the

61 visualisation instead of perusing only a set of fixed diagrams. For instance, IHME Viz Hub

62 (IHME 2017) compiles a number of datasets related to health problems around the world in

63 the form of interactive maps. Consensus PathDB (Kamburov et al. 2011) is a database that

64 integrates different types of functional interactions between physical entities in the cell and

65 uses network graphs to show the interactions between proteins. Apart from that, EcoCyc

66 (Keseler et al. 2013) is a comprehensive database outlining the genome sequence of

67 Escherichia coli, where molecular data is displayed in a detailed timeline diagram. In addition,

68 EcoCyc also allows users to customise the display to suit their interests, thus enabling them to

69 move from one region of a genome to another.

70 Another key to effective data visualisation is the combination of functioning

71 visualisation tools. A good visual library that consists of a set of programming languages 
72 helps in the design of any kind of visualisation needed. D3.js (Teller 2013) is an example of a

73 JavaScript-based library that is used for creating dynamic and interactive data visualisations in

74 a web browser. It heavily utilises Cascading Style Sheets (CSS), Hypertext Markup Language

75 (HTML), and Scalable Vector Graphics (SVG) standards, which provide controls over the

76 final result to users. D3.js is applied in visualising different types of data such as the speed and

77 direction of the wind (Cook 2017), obesity rate of adults (Map 2017), and property prices

78 (Jackson 2015).

79 A few botanic-related visualisation systems have been developed previously based on

80 different motivations. For instance, Gramene (Tello-Ruiz et al. 2016) is an open source

81 database that focuses on genomics in crops and a number of model plant species. This

82 database is curated with controlled vocabularies from a set of ontologies. Besides that, the

83 Botany Array Resource (Toufighi et al. 2005) provides web-based tools of microarray data

84 specifically for plant species and some animal species. Another example is Ensemble Plants

85 (Bolser et al. 2016), which is an online database that contains genome-level information for 39

86 plant species and functional tools for genomic alignments, functional annotation, and other

87 purposes. Apart from that, data visualisation is also used for modelling a virtual three-

88 dimensional plant model in order to simulate the growth process of crops such as the tomato

89 plant modelling system ( $\mathrm{Lu}$ et al. 2015). However, most available botanical visualisation

90 systems lack interactive elements that allow users to manipulate data, as the systems focus

91 only at the genomic level.

92 From the studies discussed above, it can be concluded that the huge number of plant

93 data is due to the wide range of plant types and species in existence, together with

94 advancements in research technology. This vast amount of data requires an effective way of 
95 presentation for both researchers and members of the community, particularly in a more

96 structured and interactive form. Presenting data in visual form helps to convey a deeper

97 meaning of the data to users, thus encouraging knowledge inference among them. Two

98 important factors that need to be considered in data visualisation are the types of visualisation

99 that best suit the data and the types of visualisation tools for development.

100 This study looks into how data visualisation can be applied in deducing relationships

101 between plant data, for example the relationship between taxonomical data, between samples

102 (i.e. physical sample of the plant), and between taxonomical data and samples. Thus, a visual

103 representation for plant data is proposed. The objectives of this study are: (1) to integrate

104 plant-based data, which consists of taxonomical data (in textual format) and samples (in

105 textual format and images); (2) to transform text-based results in XML serialization format

106 into graphical form; and (3) to develop a visualisation system for plant data. In addition, a user

107 evaluation for expert and novice users is conducted to evaluate the proposed visual 108 representation of plant data.

109

$110 \quad$ Materials \& Methods

111

112 Visual-based Representation Framework

113 The framework used in this study illustrates the flow of data representation from the database

114 that is transformed into visualisation. Fig. 1 shows a generic flowchart of the proposed visual

115 representation for plant data. There are four main steps involved: (1) conversion of the

116 ontological dataset into graph data model; (2) query from graph data model; (3)

117 transformation of text-based results in XML serialization format into graphical form; and (4)

118 display of the result to the user via a graphical user interface (GUI). 
120 Dataset

121 The dataset used in this study was obtained from Plant Ontology UM (POUM), an ontology

122 that contains tree and shrub data collected from University of Malaya (UM), Kuala Lumpur,

123 Malaysia. The following steps show the preparation of the dataset used in this study.

124 Step 1: Define the plant data description

125 Fig. 2 shows the plant data description in this study. Taxonomical classification describes the

126 rank of a plant from species to kingdom level along with the common name, authorship and

127 year. Plant morphological characteristics describe the features of the bark, leaf, fruit, flower

128 and the plant as a whole tree, at the species level. Ecological attributes describe the level of

129 water usage for plant growth, as well as the type of soil, and habitat. Geological distribution

130 describes the location and GPS coordinates of the plant sampling area.

131 In addition, the plant samples for each species were described and images of the leaf,

132 flower, bark, and fruit of the whole tree were collected as well. The morphological

133 characteristics, ecological attributes and geological distribution of plant samples were

134 described individually due to the fact that the difference of habitat environments at plant

135 sampling areas (see Fig. 3) affect the appearances of the plant samples.

137 Step 2: Build the proposed ontology

138 Plant data description as shown in Fig. 2 was then translated into the proposed ontology

139 schema as shown in Fig. 4. The concepts (the entity), the concepts' properties (the data

140 property) and the relationships between concepts (the object property) of the domain were

141 determined in this schema. 
To prevent any misunderstanding in the terms used to describe the plant, a

143 standardized set of vocabularies was designed. These vocabularies were adapted from the

144 existing schema, namely the Biodiversity Information Standards (TDWG, 2018) which

145 consists of a number of specific biodiversity data standards such as Life Sciences Identifier

146 (LSID), Darwin Core, and TDWG Access Protocol for Information Retrieval (TAPIR).

147 Besides that, few vocabularies were also newly defined because these vocabularies are

148 unavailable in any existing schema.

149 This ontology schema was then converted into an ontological form, a machine-

150 readable formal specification (in owl file format) which proceeded to the reasoning process to

151 complete the process of plant data annotation. Protégé 5.2 was used for these purposes. Fig. 5

152 shows a partial of POUM using OntoGraph plug-in in Protégé 5.2.

154 There are more than 200 images of plants in the image database. These images are annotated 155 with vocabularies from the POUM, consisting of 8 main classes, 5 sub-classes, 17 object 156 properties, and 39 data properties (Table S1). There are 43 species of 42 genera for trees and 15731 species of 28 genera for shrubs (Table S2 and Table S3) with a total of 222 samples. As 158 shown in Fig. 6, the image of a plant sample is described using sample information such as 159 attributes of the sample's bark, leaf, flower, and fruit, object of the image, location of where 160 the sample was collected, and taxonomic data that focuses on taxonomical ranks and plant 161 characteristics used in describing the plant species.

164 Fig. 7 shows the workflow of passing ontological data into the creation of a network graph. 
165 Step 1: Conversion of ontological dataset into a graph data model

166 Ontological data of the POUM in owl file format is commonly encoded in a Resource

167 Description Framework (RDF) data model with Extensible Markup Language (XML) syntax.

168 Apache Jena (Jena 2017) introduced the serialisation of ontological data into an RDF graph

169 data model before it was queried.

170

171 Step 2: Query from graph data model

172 The query languages used were Simple Protocol and RDF Query Language (SPARQL). RDF

173 query language retrieves and manipulates any data stored in RDF format (Harris et al. 2013).

174 Once users submit a text query, an SPARQL query is sent to the server, where querying is 175 executed to the RDF graph data model of the POUM. The results of the query are in the form 176 of XML syntax and then further structured into a JSON format text to be used by the D3.js 177 library.

Step 3: Transformation of text-based results in XML serialization format into graphical form

180 The relationship between data is crucial for highlighting data association in the knowledge 181 domain. Three types of relationships are taken into consideration:

182 1. The relationship between one taxon to another taxon. A taxon is linked to another by 183 its family name. For example, Delonix regia and Acacia auriculiformis are linked to one another as both are in the same family, Fabaceae. 
185

2. The relationship between a taxa and its sample. Each taxon has three samples and each sample has a unique identifier. For instance, Murraya paniculata has three samples, namely 'SMurPan001', 'SMurPan002', and 'SMurPan003'.

3. The relationship between samples. Samples are related when they are from the same taxon or are obtained from the same location. For instance, samples of Lagerstroemia indica, Manihot esculenta, and Terminalia catappa are collected from the same location, which is 'DTC UM'.

These relationships can be clearly shown when illustrated to users in a visual form. In order to transform texts into a graphical form, the D3.js visualisation library was chosen, as this utilises different types of programming languages in the data visualisation design. The library aims to shorten the development process while maintaining the quality of the system. There are many types of visualisations that can be designed using D3.js; thus, it is important to choose a suitable type of visualisation.

For the type of visualisation in this study, a network graph was chosen. This graph enables the visualisation of plant data, as the relationship between one data to another can be illustrated clearly. The network graph is a type of graph that highlights the relationship between entities and consists of 'nodes' as entities and 'links' as lines to link between entities. There are two types of nodes, which are the 'parent' $(\mathrm{PN})$ and 'children' $(\mathrm{CN})$ nodes. PN is a type of node that has one or more $\mathrm{CN}$. Meanwhile, $\mathrm{CN}$ is a type of node that has a PN ancestor.

Before generating the graphic representation, a canvas was first set up. The D3.js library uses a layout controlled by force and SVG as the container for the visualisation. A few parameters were defined such as distance, gravity, and size from the function 
208 d3.layout.force(). The result in JSON format is called a variable, as it was further arranged 209 using D3.js to create a network graph. Besides that, some interactive features such as view 210 node label, highlight node's links, expand or shrink group of nodes, page of sample 211 information, and thumbnail images of plant sample were added into the network graph in 212 order to develop a user-friendly system. This is to enable the interaction between user and 213 data. Hence, users were able to manipulate the content of the network graph with the 214 implemented features.

215

216 Step 4: Display result to the user in a graphical user interface (GUI)

217 The generated network graph was then displayed in the GUI to provide the full experience to 218 users, as this was embedded in the interface.

Data and source code for the PlantViz development can be accessed and retrieved from

221 https://github.com/afrinaad/PlantViz/.

223 User Testing

224 There are a variety of data visualisation tools available to help developers achieve the 225 objective of data visualisation. Therefore, it is desirable to determine whether or not the 226 developed visualisation is successful in achieving user needs. In order to measure the 227 performance of the developed plant data visualisation, a user evaluation was carried out. User 228 evaluation consists of two tests, namely: (1) usability heuristics and (2) query and 229 visualisation evaluation. User evaluation involved expert users with research background or 230 experience in the botanical field including users that might have little skill in information 
231 technology (IT). Meanwhile, novice users who have little or no research background or 232 experience in the botanical field might have skills in IT.

233 The usability heuristic evaluation was adapted from Nielsen's 10 usability heuristics 234 for user interface (UI) design (Nielsen 1992). These are the general principles for an 235 interactive UI design. In this study, 10 usability features were adapted to match the developed 236 visualisation system. A sample of the questionnaire used in this study is shown in Fig. 8. 237 Users were given five minutes to explore the GUI before the evaluation. This step was 238 performed to observe the users' first impression of the visualisation system.

239 A query and visualisation evaluation was conducted to assess the efficiency of the 240 visualisation system in delivering visualised content to users (Amri et al. 2015; Hearst et al. 241 2016). This helps measure the performance of query sending from the GUI to the server and 242 translates the results into a visual format. In addition, this evaluation assists in the observation 243 of the ability of users to interact with data successfully using features of the visualisation 244 system. Fig. 9 shows a sample of the questionnaire for this purpose. Users were given 245 guidelines and instructions for using the developed data visualisation system before 246 performing user evaluation. Both the guidelines and instructions were given to four different 247 cases based on specific search parameters, namely Scientific Name (Case 1), Family Name 248 (Case 2), Location (Case 3), and Water Usage (Case 4). Query and visualisation were 249 evaluated via a rating of ' 1 ' to '5', where '1'-Poor, '2'-Fair, '3'-Average, '4'-Good, and '5'250 Excellent. Any comment from the users was taken into consideration in order to improve the 251 developed visualisation system.

252 Next, Fisher's Exact test and t-test were performed to analyse the outcome of the 253 evaluation. Based on the usability heuristic evaluation, Fisher's Exact was conducted to check 
254 whether or not the GUI of the system is dependent on the users' knowledge in botanic and IT.

255 Meanwhile, based on the query and visualisation evaluation, a t-test was conducted to check

256 whether or not there was any significant difference between the evaluation done by expert and

257 novice users on the visualization system.

259 Results

260 PlantViz - plant visualisation system

261 Based on the proposed visual-based representation for plant species data, a prototype of the

262 web-based plant data visualisation system was developed called PlantViz, as shown in Fig.

263 10. PlantViz consists of a query tool to search for data in POUM and a graphical viewer to

264 display the retrieved results to users. There are four parameters in the Query page, which are 265 based on Scientific Name, Family Name, Location, and Water Usage. The Scientific Name and 266 Family Name parameters are commonly used as search parameters in many public databases 267 (NRCS 2017; PFAF 2017; UCONN 2017). Besides that, the Location parameter is for the 268 location where the plant sample was collected. This was chosen as one of the query 269 parameters because the plant samples in this study were mainly collected from various areas 270 in University of Malaya. In addition, PlantViz's target users are members of the university, 271 who are familiar with these locations such as 'DTC UM', 'Tasik Varsiti UM', and 'Fakulti 272 Sains UM,' all locations in University of Malaya. The Water Usage parameter defines the 273 level of water needed by a plant species for its growth. Since parameters such as Scientific 274 Name and Family Name were used to represent the taxonomical aspect and Location was used 275 to represent the geographical information of the plant. The Water Usage parameter was 276 chosen to represent the morphology attribute of the plant. 
The proposed methodology for transforming text-based results into visualisation form

278 was implemented in PlantViz. Fig. 11 shows a fragment of the ontological data serialised into

279 an RDF graph data model that contains a collection of RDF nodes attached to each other by

280 annotated relations. This process was done using a ModelFactory class from the Apache Jena

281 library.

282 The RDF graph data model was then queried from when the users used the query tool

283 in PlantViz. Textual query from users was sent as a SPARQL query using QueryFactory and

284 QueryExecutionFactory classes from Apache Jena. The result of the query was in XML

285 format, which was then restructured into JSON format. The flexibility of JSON enables users

286 to organise results according to any arrangement, as JSON can contain any number, Unicode

287 character, Boolean operators, array, object, or null value (Bassett 2015). Fig. 12 shows an

288 example of the query results in both XML and JSON formats. The example shown is a

289 shortened part of the result to show the difference in terms of structure for the query result

290 before and after being transformed into JSON. Fig. 12(a) illustrates the results generated by

291 Apache Jena, which is in XML format. This was then rearranged into JSON format and later

292 organised into different arrays based on the relationship of the data, as shown in Fig. 12(b).

293 For example, general information on the plant such as its common name, water usage, and soil

294 type were organised into the same array. Results of the query in JSON format were then

295 passed to the D3.js library to form a network graph as a visualisation of the query result. For

296 instance, the network graph shown in Fig. 13 is the result of querying the Magnolia figo plant 297 species.

298 Three types of data relationships, as mentioned earlier, are clearly shown from the 299 results of each chosen query parameter and depicted in Fig. 14. Moreover, unlike Plant 
300 Ontology (Jaiswal et al. 2005), which provides a static graphical view, the graphical viewer in 301 PlantViz provides interactive elements, which allow users to explore the result (Lohmann et 302 al. 2015), as illustrated in Fig. 15. Table 1 lists the description of visualisation features, 303 functions, and its conditions. PlantViz is freely accessible at http://103.18.1.10:8080/plantviz/. The detailed manual 305 on how to use the PlantViz is provided as supplemental Article S1.

\section{User Evaluation}

308 User evaluation was performed on the querying and visualisation of PlantViz. 60 users 309 including 30 expert users and 30 novice users participated in this user evaluation. The expert 310 users are botanists and researchers in biodiversity with little IT knowledge while the novice 311 users are undergraduate students from University of Malaya.

312 Fig. 16 presents an analysis of the usability heuristics evaluation by both expert and 313 novice users. This shows that the majority of users rated 'Yes' for most of the features. As 314 shown in Fig. 16(a), E6 has the highest number for the rating 'Yes' (all 30 expert users voted 315 'Yes'), while for novice users, as shown in Fig. 16(b), E2, E6, and E9 have the highest 316 number for the rating 'Yes' (all 30 novice users voted 'Yes'). Meanwhile, for both types of 317 users, E1 has the highest number for rating 'No' (16 out of 30 expert users and 14 out of 30 318 novice users voted 'No'). This is consistent with prototype development. The guidelines for 319 using the system are available with no status for the system being shown.

320 Fisher's Exact test was conducted to check whether or not the user experience in using 321 PlantViz is dependent on their expertise level. The null hypothesis $\mathrm{H}_{0}$ is that there is no 322 difference between the usability heuristics evaluation performed between expert users and 
323 novice users. The two-tailed probability $(p)$ value of Fisher's Exact test on usability heuristics

324 evaluation is $0.312,(p<0.05)$, which means that there is no significant difference in the

325 usability heuristic evaluation between expert and novice users. This also indicates that

326 PlantViz's user interface is adequate for all types of users regardless of their IT knowledge.

327 Analyses of query and visualisation evaluation for all four cases by both expert and

328 novice users are shown in Fig. S1 and Fig. S2, respectively. Fig. 17 shows the analysis of

329 query evaluation by expert and novice users. The total number of responses for each case is

330 plotted against the evaluation rating. The query evaluation by both types of users is shown in

331 Fig. 17(a) and Fig. 17(b). These show similar results whereby the majority of expert and

332 novice users gave a '4' or '5' rating for most of the query criteria. There is one response that

333 gave a rating of ' 1 ' (one expert user) and a total of nine responses that gave a rating of ' 2 ' (six

334 expert and three novice users). A low rating was given for $Q 4$, as shown in Fig. S1.

335 Furthermore, Fig. 18 shows the analysis of visualisation evaluation by expert and

336 novice users. The total number of responses for each case is plotted against the evaluation

337 rating as well. Fig. 18(a) and Fig. 18(b) show that both types of users rated '4' for most of the

338 visualisation criteria. There is one response with a rating of ' 1 ' and 35 responses with a rating

339 of '2' (see Fig. S2). $V 7$ was given a rating of '1' (one expert user rated '1') and had the

340 highest number for a rating of ' 2 ' (four experts and nine novice users rated ' 2 '). It can

341 therefore be concluded that the PlantViz interactive feature for the sample information page

342 should be improved and be more easily accessible. Besides that, $V 6$ had seven responses with

343 a rating of ' 2 ' (two experts and five novice users rated '2'), which shows that users did not

344 agree that the information shown in the graphical viewer was sufficient. More information

345 could be added to the network graph such as type of life cycle, propagation method, and 
346 characteristics of other parts of plants. Linking the data to other external databases such as the

347 accession term in Trait Ontology (Walls et al. 2012) and Plant Ontology would extend the

348 information scope to users.

349 Independent sample t-tests were conducted to test the significance of the evaluation

350 done by both types of users. The null hypothesis $\mathrm{H}_{0}$ declares that there is no difference

351 between the evaluation of expert and novice users. T-tests were performed on each case for

352 both evaluations with results as shown in Table 2. The $p$-values for all cases are higher than

353 the significance level, thus there is a lack of evidence to reject $\mathrm{H}_{0}$. Hence, it can be concluded 354 that there is no statistically significant difference between the query and visualisation 355 evaluation performed by expert and novice users. This signifies that PlantViz can be used by 356 users of all levels, with or without knowledge in the botanical or IT fields.

\section{Discussion}

359 Many plant species share resemblance in appearance, yet there are many characteristics such

360 as genomic data, morphological attributes, and geographical attributes that can distinguish one

361 plant species from the other. More often than not, the data or information in textual form

362 found on many online databases are presented using lengthy and wordy descriptions.

363 Consequently, this hinders researchers from deducing new knowledge from the presented

364 plant data. In this paper, we report an alternative approach for presenting plant data, which is

365 via network graph. Thus, by visualising the retrieved results from the database, the problem of

366 lengthy retrieved texts as part of the results can be eliminated. In addition, this method

367 emphasises the relationship between data and presents this information in a visualised form

368 for users. Hence, from the visualised data, the relationship between data can be easily 369 analysed and inferred. 
371 can use PlantViz via a user-friendly graphical user interface (GUI). Thus, two types of tests

372 were conducted to assess the performance efficiency of PlantViz, namely usability heuristics

373 evaluation and query and visualisation evaluations.

374 From the observation, the $p$-values of the query and visualisation evaluation (Table 2)

375 share the same pattern, with Case 1 having the lowest $p$-value. It can be presumed that in Case

376 1, users were not yet familiar with the graphical viewer of the PlantViz system. This is

377 because the graphical viewer is not a common tool in many public plant-based databases such

378 as The Plant List (List 2013) and NParks Flora \& Fauna (Board 2013). However, all other

379 cases namely Case 2, Case 3, and Case 4 have $p$-values higher than Case 1 for both

380 evaluations; hence, the previous assumption is valid, as users had just started to become

381 acquainted with PlantViz. In addition, the GUI design of both the query tool and graphical

382 viewer in PlantViz is simple yet still appropriate for both types of users. As for Case 4, both

383 evaluations had the highest $p$-value. Therefore, it can be concluded that users were easily

384 accustomed to the PlantViz system. This also verifies that the GUI for PlantViz is consistent

385 throughout all cases, whereby each case uses different search parameters.

386 Yet, filtering feature could be added to the graphical viewer to grant users the ability to

387 filter search results. For instance, the Location parameter was used as the search parameter for

388 Case 3 and this could generate a cluttered network graph, as many plant species were

389 collected from the same location. Thus, as mentioned by Cline et al. (2007), users could filter

390 the results by selecting only a certain type as shown in the graphical viewer, and this could

391 help in retrieving the most relevant one. In addition, users should be allowed to filter other 
392 types of search parameters such as family name, number of plant sample collected, or type of 393 plant.

394 The query method described here is simple — a typical Boolean search is used. Thus, a 395 more robust and efficient query could be achieved by implementing other query and search 396 methods such as the ranking algorithm (Tran et al. 2009; Zhiguo \& Zhengjie 2010) and 397 natural language query processing (Damljanovic et al. 2010; Paredes-Valverde et al. 2015; 398 Tablan et al. 2008; Varga et al. 2014; Wang et al. 2007). It is vital for users to query the 399 system without being attached to a fixed one and to select pre-defined parameters. Based on 400 the low-rated $Q 4$, as shown in Fig. S1, users were dissatisfied with the number of search 401 parameters that can be used to query at a time. This limitation will be enhanced in the future 402 by allowing users to add more than one search parameter to narrow down the retrieved results, 403 so that more relevant results could be retrieved.

404 The POUM currently consists of plant descriptions and their samples in textual and 405 image forms. PlantViz was able to visualise the POUM dataset by showing the relationships 406 between: (1) 'taxon-taxon'; (2) 'taxon-sample'; and (3) 'sample-sample'. This dataset will be 407 extended to include other plant types along with their descriptions and plant systematics, 408 behaviour, ecology, and diversity. Thus, POUM could be linked (Hebeler et al. 2009; Smith et 409 al. 2007) as well to other existing ontologies (Tello-Ruiz et al. 2016). For this reason, PlantViz 410 will be further enhanced to support additional features such as: (1) querying from different 411 datasets at a time and show their relationships, and (2) providing data analytics by inferring 412 the visualised relationships.

413 Furthermore, the visualisation feature will also be improved in order to enhance the 414 usability of the proposed visual-based representation for plant data. Other features such as 
415 enabling users to choose the types of visualisation to be generated and choosing a colour 416 scheme to differentiate the relationships between data could be considered as well.

417 In addition, it is possible to apply the visual-based representation for large dataset such 418 as PlantCLEF (Goeau et al. 2017) and National Phenology Database (Marsh 2017) as these 419 two examples have the database backend and provide the visualization tool. Definitely, the 420 data will be retrieved; however the visualised data will be cluttered and interactivity might be 421 inefficient. Hence, an extra task, pre-processing the retrieved data such as through 422 dimensionality reduction and data pre-fetch may be needed before the data could be 423 visualised. This pre-processing would retrieve a lesser amount of data, whereby 424 dimensionality reduction helps in reducing the computational load (Kaski \& Peltonen 2011), 425 while data pre-fetch helps in improving the response time (Battle et al. 2016). Besides that, 426 this method could be improved by employing the hierarchical exploration method which is 427 commonly used to visualize large and high dimensional data as proposed by Lin et al (2013), 428 Yang et al. (2003) and Zinsmaier et al. (2012). This method is practical because it allows data 429 visualization in different levels of details (Bikakis 2018).

\section{Conclusion}

432 The field of biology generates thousands of data every day with the advancements in modern 433 tools and technologies. Hence, it is important to implement a proper methodology for 434 retrieving data and making it accessible to users in an effective way. This study focuses on 435 presenting retrieved data from an ontology to users in a visualisation form. Hence, a visual 436 representation of plant data, PlantViz, was proposed. Data from POUM database was 437 converted as a graph data model and queried using SPARQL. Results from the query were 438 then structured into JSON format before being transformed into a visualisation form and then 
439

440

441

442

443

444

445 446 insights.

presented to users in GUI form. User evaluation analysis and results show that PlantViz can be used by users of different levels, either expert users from the botanical field, students, or laymen with interest in botany. PlantViz eliminates lengthy texts of information as results for user queries. In addition, users were able to interact with the data directly, as the visualisation is dynamic. Most importantly, this technique demonstrates the practicability of using computer-assisted tools by providing cognitive analysis to understand the relationship between data. Moreover, this study also facilitates users in inferring and gaining new data

\section{References}

Amri S, Ltifi H, and Ayed MB. 2015. Towards an intelligent evaluation method of medical data visualizations. 2015 15th International Conference on Intelligent Systems Design and Applications (ISDA).

Armstead I, Huang L, Ravagnani A, Robson P, and Ougham H. 2009. Bioinformatics in the orphan crops. Briefings in Bioinformatics 10. 10.1093/bib/bbp036

Bassett L. 2015. Introduction to JavaScript Object Notation: A To-the-Point Guide to JSON: O'Reilly Media.

Battle L, Chang R, and Stonebraker M. 2016. Dynamic Prefetching of Data Tiles for Interactive Visualization. Proceedings of the 2016 International Conference on Management of Data. San Francisco, California, USA: ACM. p 1363-1375.

Bikakis N. 2018. Big Data Visualization Tools: Springer.

Board NP. 2013. NParks Flora\&FaunaWeb. Available at https://florafaunaweb.nparks.gov.sg/Home.aspx (accessed 7 February 2018).

Bolser D, Staines DM, Pritchard E, and Kersey P. 2016. Ensembl Plants: Integrating Tools for Visualizing, Mining, and Analyzing Plant Genomics Data. In: Edwards D, ed. Plant Bioinformatics: Methods and Protocols. New York, NY: Springer New York, 115140.

Chen CM, Lai KJ, Pai TW, and Chang HT. 2014. Transcriptome Data Visualization in Pathways with Application to Zebrafish Embryo Datasets. 2014 Eighth International Conference on Complex, Intelligent and Software Intensive Systems (CISIS): IEEE. p 515-518.

Cline MS, Smoot M, Cerami E, Kuchinsky A, Landys N, Workman C, Christmas R, AvilaCampilo I, Creech M, Gross B, Hanspers K, Isserlin R, Kelley R, Killcoyne S, Lotia S, Maere S, Morris J, Ono K, Pavlovic V, Pico AR, Vailaya A, Wang PL, Adler A, Conklin BR, Hood L, Kuiper M, Sander C, Schmulevich I, Schwikowski B, Warner GJ, Ideker T, and Bader GD. 2007. Integration of biological networks and gene expression data using Cytoscape. Nat Protoc 2:2366-2382. 10.1038/nprot.2007.324 
476

477

478

479

480

481

482

483

484

485

486

487

488

489

490

491

492

493

494

495

496

497

498

499

500

501

502

503

504

505

506

507

508

509

510

511

512

513

514

515

516

517

518

519

520

521

Cook P. 2017. UK Wind Chart. Available at http://charts.animateddata.co.uk/ukwind/ (accessed 28 October 2017).

Damljanovic D, Agatonovic M, and Cunningham H. 2010. Natural Language Interfaces to Ontologies: Combining Syntactic Analysis and Ontology-Based Lookup through the User Interaction. Berlin, Heidelberg: Springer Berlin Heidelberg. p 106-120.

Groenendyk M. 2013. Emerging Data Visualization Technologies for Map and Geography Libraries: 3-D Printing, Holographic Imaging, 3-D City Models, and 3-D Model-based Animations. Journal of Map \& Geography Libraries 9. $10.1080 / 15420353.2013 .821436$

Goeau H, Bonnet P, and Joly A. 2017. Plant identification based on noisy web data: the amazing performance of deep learning (LifeCLEF 2017). CLEF 2017-Conference and Labs of the Evaluation Forum. p 1-13.

Harris S, Seaborne A, and Prud'hommeaux E. 2013. SPARQL 1.1 query language. Available at https://www.w3.org/TR/rdf-sparql-query/ (accessed 21 October 2017).

Hearst MA, Laskowski P, and Silva L. 2016. Evaluating Information Visualization via the Interplay of Heuristic Evaluation and Question-Based Scoring. Proceedings of the 2016 CHI Conference on Human Factors in Computing Systems. San Jose, California, USA: ACM. p 5028-5033.

Hebeler J, Fisher M, Blace R, and Perez-Lopez A. 2009. Semantic Web Programming: Wiley Publishing.

Hughes S. 2006. Opinion piece: Genomics and crop plant science in Europe. Plant Biotechnology Journal 4. 10.1111/j.1467-7652.2005.00164.x

IHME. 2017. Life Expectancy and Probability of Death | IHME Viz Hub. Available at http://vizhub.healthdata.org/le/ (accessed 3 March 2017 2017).

Jackson H. 2015. E\&W Property Price Map. Available at http://helenjacksonanalytic.co.uk/EW prop price.html (accessed 28 October 2017).

Jaiswal P, Avraham S, Ilic K, Kellogg EA, McCouch S, Pujar A, Reiser L, Rhee SY, Sachs MM, Schaeffer M, Stein L, Stevens P, Vincent L, Ware D, and Zapata F. 2005. Plant Ontology (PO): a Controlled Vocabulary of Plant Structures and Growth Stages. Comparative and Functional Genomics 6. 10.1002/cfg.496

Jena A. 2017. A free and open source Java framework for building Semantic Web and Linked Data applications. Available at https://jena.apache.org/ (accessed 28 October 2017).

Jensen PA, and Papin JA. 2014. MetDraw: automated visualization of genome-scale metabolic network reconstructions and high-throughput data. Bioinformatics 30 . 10.1093/bioinformatics/btt758

Kamburov A, Pentchev K, Galicka H, Wierling C, Lehrach H, and Herwig R. 2011. ConsensusPathDB: toward a more complete picture of cell biology. Nucleic acids research 39. 10.1093/nar/gkq1156

Kaski S, and Peltonen J. 2011. Dimensionality Reduction for Data Visualization [Applications Corner]. IEEE Signal Processing Magazine 28:100-104. 10.1109/MSP.2010.940003

Keim DA. 2002. Information Visualization and Visual Data Mining. IEEE Transactions on Visualization and Computer Graphics 8:8. 10.1109/2945.981847

Keseler IM, Mackie A, Peralta-Gil M, Santos-Zavaleta A, Gama-Castro S, BonavidesMartinez C, Fulcher C, Huerta AM, Kothari A, Krummenacker M, Latendresse M, Muniz-Rascado L, Ong Q, Paley S, Schroder I, Shearer AG, Subhraveti P, Travers M, Weerasinghe D, Weiss V, Collado-Vides J, Gunsalus RP, Paulsen I, and Karp PD. 
2013. EcoCyc: fusing model organism databases with systems biology. Nucleic Acids Res 41. 10.1093/nar/gks1027

List TP. 2013. The Plant List - A working list of all plant species. Available at http://www.theplantlist.org/ (accessed 7 February 2018).

Lin Z, Cao N, Tong H, Wang F, Kang U, and Chau DHP. 2013. Demonstrating Interactive Multi-resolution Large Graph Exploration. 2013 IEEE 13th International Conference on Data Mining Workshops. p 1097-1100.

Lohmann S, Link V, Marbach E, and Negru S. 2015. WebVOWL: Web-based Visualization of Ontologies. Cham: Springer International Publishing. p 154-158.

Lu C, Deng L, and Fei M. 2015. An improved visualization modelling method of greenhouse tomato plants based on L-system. Chinese Automation Congress (CAC), 2015: IEEE. p 480-485.

Map V. 2017. US Map States - Choropleth Plus Bar. Available at https://vida.io/documents/4vZ9mRGyepoyQxFcK (accessed 28 October 2017).

Marsh L. 2017. The USA National Phenology Network: A Framework for Delivery of Phenology Data Products on Multiple Spatiotemporal Scales. Biodiversity Information Science and Standards 1:e20188.

Marx V. 2013. Biology: The big challenges of big data. Nature 498:255-260. $10.1038 / 498255 \mathrm{a}$

Nielsen J. 1992. Finding usability problems through heuristic evaluation. Proceedings of the SIGCHI conference on Human factors in computing systems: ACM. p 373-380.

NRCS U. 2017. The PLANTS Database. National Plant Data Team, Greensboro.

Paredes-Valverde MA, Rodríguez-García MÁ, Ruiz-Martínez A, Valencia-García R, and Alor-Hernández G. 2015. ONLI: An ontology-based system for querying DBpedia using natural language paradigm. Expert Systems with Applications 42. 10.1016/j.eswa.2015.02.034

PFAF. 2017. The PFAF Database (Plant For A Future).

Sedova M, Jaroszewski L, and Godzik A. 2016. Protael: protein data visualization library for the web. Bioinformatics 32:602-604. 10.1093/bioinformatics/btv605

Smith B, Ashburner M, Rosse C, Bard J, Bug W, Ceusters W, Goldberg LJ, Eilbeck K, Ireland A, Mungall CJ, Leontis N, Rocca-Serra P, Ruttenberg A, Sansone S-A, Scheuermann RH, Shah N, Whetzel PL, and Lewis S. 2007. The OBO Foundry: coordinated evolution of ontologies to support biomedical data integration. Nat Biotech 25:1251-1255. 10.1038/nbt1346

Tablan V, Damljanovic D, and Bontcheva K. 2008. A Natural Language Query Interface to Structured Information. In: Bechhofer S, Hauswirth M, Hoffmann J, and Koubarakis M, eds. The Semantic Web: Research and Applications: 5th European Semantic Web Conference, ESWC 2008, Tenerife, Canary Islands, Spain, June 1-5, 2008 Proceedings. Berlin, Heidelberg: Springer Berlin Heidelberg, 361-375.

TDWG, B. I. S. (2018). Biodiversity Information Standards (TDWG). Retrieved 25th March 2018 from https://github.com/tdwg

Tegarden DP. 1999. Business information visualization. Communications of the AIS 1:4.

Telea AC. 2014. Data Visualization: Principles and Practice, Second Edition: A. K. Peters, Ltd.

Teller S. 2013. Data Visualization with d3.js: Packt Publishing. 
567

568

569

570

571

572

573

574

575

576

577

578

579

580

581

582

583

584

585

586

587

588

589

590

591

592

593

594

595

596

597

598

599

600

601

602

603

604

Tello-Ruiz MK, Stein J, Wei S, Preece J, Olson A, Naithani S, Amarasinghe V, Dharmawardhana P, Jiao Y, Mulvaney J, Kumari S, Chougule K, Elser J, Wang B, Thomason J, Bolser DM, Kerhornou A, Walts B, Fonseca NA, Huerta L, Keays M, Tang YA, Parkinson H, Fabregat A, McKay S, Weiser J, D'Eustachio P, Stein L, Petryszak R, Kersey PJ, Jaiswal P, and Ware D. 2016. Gramene 2016: comparative plant genomics and pathway resources. Nucleic Acids Res 44. 10.1093/nar/gkv1179

Tory M, and Moller T. 2004. Human factors in visualization research. IEEE transactions on visualization and computer graphics 10:72-84. 10.1109/TVCG.2004.1260759

Toufighi K, Brady SM, Austin R, Ly E, and Provart NJ. 2005. The Botany Array Resource: eNortherns, Expression Angling, and promoter analyses. The Plant Journal 43:153-163. 10.1111/j.1365-313X.2005.02437.x

Tran VX, Tsuji H, and Masuda R. 2009. A new QoS ontology and its QoS-based ranking algorithm for Web services. Simulation Modelling Practice and Theory 17. 10.1016/j.simpat.2009.06.010

UCONN. 2017. UCONN Plant Database. University of Conneticut.

Varga B, Trambitas-Miron AD, Roth A, Marginean A, Slavescu RR, and Groza A. 2014. LELA - a natural language processing system for Romanian tourism. 2014 Federated Conference on Computer Science and Information Systems. Warsaw, Poland: IEEE.

Walls RL, Athreya B, Cooper L, Elser J, Gandolfo MA, Jaiswal P, Mungall CJ, Preece J, Rensing S, Smith B, and Stevenson DW. 2012. Ontologies as integrative tools for plant science. Am J Bot 99:1263-1275. 10.3732/ajb.1200222

Wang C, Xiong M, Zhou Q, and Yu Y. 2007. PANTO: A Portable Natural Language Interface to Ontologies. In: Franconi E, Kifer M, and May W, eds. The Semantic Web: Research and Applications: 4th European Semantic Web Conference, ESWC 2007, Innsbruck, Austria, June 3-7, 2007 Proceedings. Berlin, Heidelberg: Springer Berlin Heidelberg, 473-487.

Willis KJ. 2017. State of the World's Plants 2017. Report Royal Botanic Gardens, Kew.

Yang J, Ward MO, and Rundensteiner EA. 2003. Hierarchical Exploration of Large Multivariate Data Sets. In: Post FH, Nielson GM, and Bonneau G-P, eds. Data Visualization: The State of the Art. Boston, MA: Springer US, 201-212.

Yuk M, and Diamond S. 2014. Data Visualization for Dummies: John Wiley \& Sons, Inc.

Zhiguo D, and Zhengjie D. 2010. Improved ontology ranking algorithm based on semantic web. 2010 3rd IEEE International Conference on Ubi-Media Computing. Jinhua, China: IEEE.

Zinsmaier M, Brandes U, Deussen O, and Strobelt H. 2012. Interactive Level-of-Detail Rendering of Large Graphs. IEEE Transactions on Visualization and Computer Graphics 18:2486-2495. 10.1109/TVCG.2012.238 


\section{Table $\mathbf{1}$ (on next page)}

List of visualisation features in PlantViz.

The features' description, function, and its conditions. 
1 Table 1. Description of visualisation features in PlantViz

\begin{tabular}{|c|c|c|}
\hline Visualisation features & Functions & Conditions \\
\hline $\begin{array}{l}\text { View node label (Fig. } \\
\text { 15a) }\end{array}$ & To show the label of a node & - Visible when the cursor is hovered \\
\hline $\begin{array}{l}\text { Highlight node's links } \\
\text { (Fig. 15b) }\end{array}$ & $\begin{array}{l}\text { To show relationship between } \\
\text { nodes }\end{array}$ & $\begin{array}{l}\text { - Visible when the cursor is hovered } \\
\text { - Only highlights links connected to } \\
\text { the node }\end{array}$ \\
\hline $\begin{array}{l}\text { Expand or shrink group } \\
\text { of nodes (Fig. 15c) }\end{array}$ & $\begin{array}{l}\text { To allow users to expand or } \\
\text { shrink a group of nodes }\end{array}$ & $\begin{array}{l}\text { - When users click on node of 'parent' } \\
\text { type } \\
\text { - Only node of 'parent' type can be } \\
\text { expanded or shrunk }\end{array}$ \\
\hline $\begin{array}{l}\text { Sample information page } \\
\text { (Fig. 15d) }\end{array}$ & $\begin{array}{l}\text { To redirect users to a new page } \\
\text { containing information of } \\
\text { plant samples }\end{array}$ & $\begin{array}{l}\text { - When node with text 'More detail' is } \\
\text { clicked }\end{array}$ \\
\hline $\begin{array}{l}\text { Thumbnail images of } \\
\text { plant samples (Fig. 15e) }\end{array}$ & $\begin{array}{l}\text { To show thumbnail-sized } \\
\text { images of plant samples }\end{array}$ & $\begin{array}{l}\text { - Visible when the cursor is hovered } \\
\text { on nodes with label 'Sample ID' }\end{array}$ \\
\hline
\end{tabular}

2 


\section{Table 2 (on next page)}

t-test results.

Results of independent sample t-test for query and visualisation evaluation. 
1 Table 2. Results of independent sample t-test for query and visualisation evaluation

\begin{tabular}{|c|c|c|}
\hline \multirow{2}{*}{ Cases } & \multicolumn{2}{|c|}{$p$-value } \\
\cline { 2 - 3 } & Query evaluation & Visualisation evaluation \\
\hline Case 1 & 0.082 & 0.133 \\
\hline Case 2 & 0.105 & 0.165 \\
\hline Case 3 & 0.177 & 0.172 \\
\hline Case 4 & 0.225 & 0.409 \\
\hline
\end{tabular}

2 
Figure 1

A proposed visual-based representation framework.

Generic flowchart of plant data visualisation.

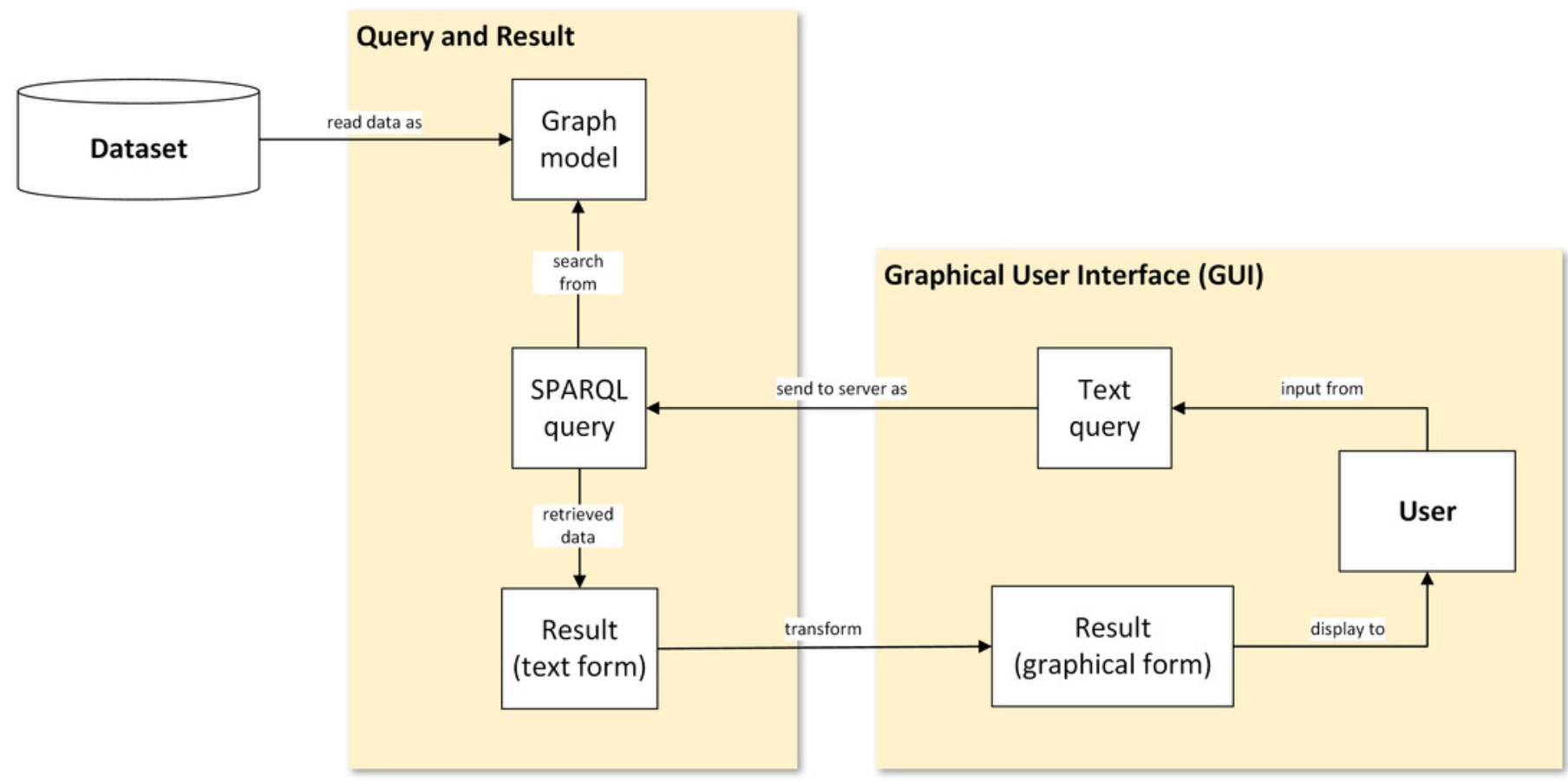


Figure 2

Plant data description.

A plant is described with the taxonomical classification, morphological characteristics, ecological attributes, geological distribution, and the plant images. Photo credit: The photo archive at http://103.18.1.10:8080/plantviz/.

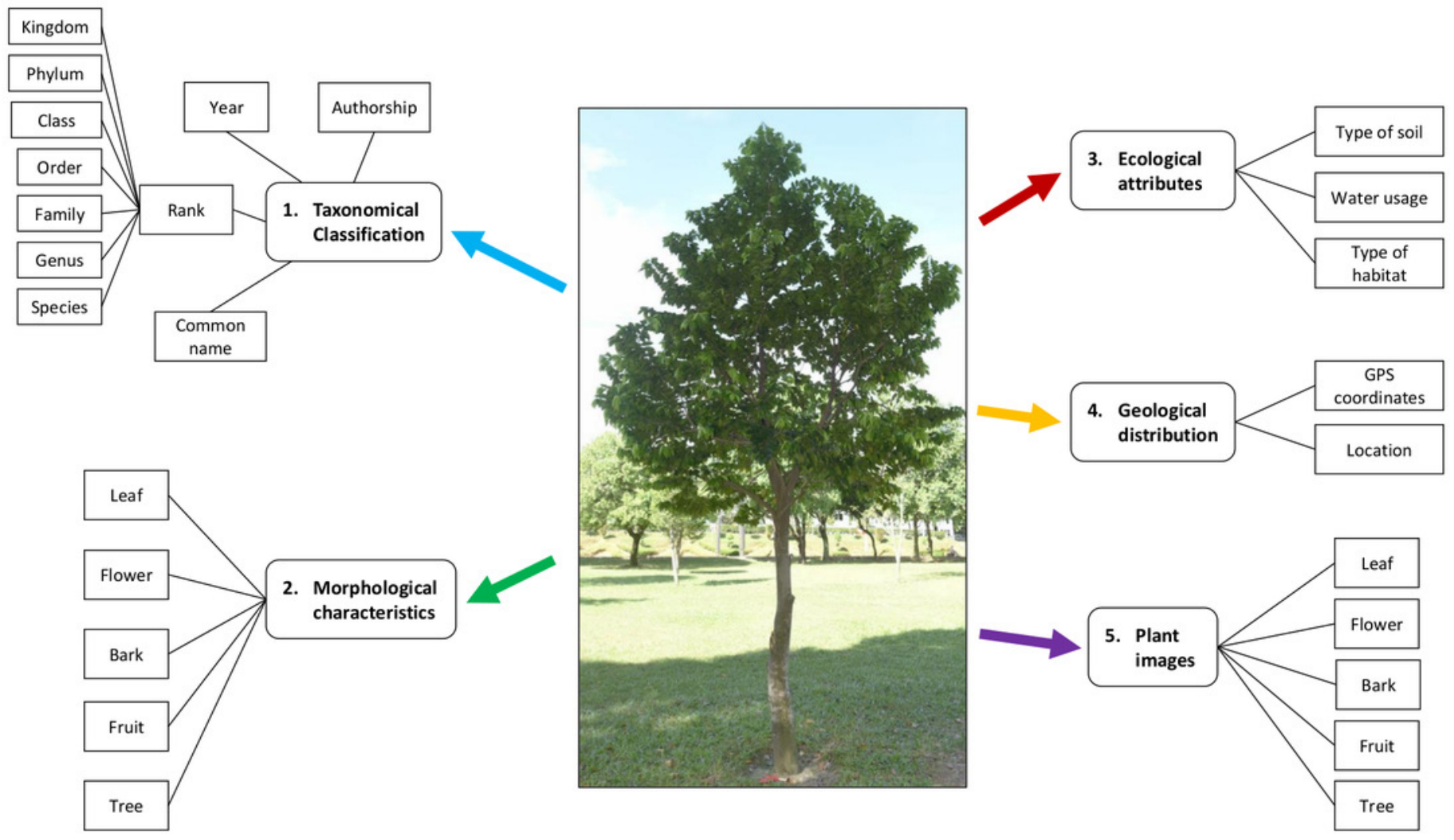


Figure 3

Plant sampling areas in UM.

There are five main locations which are DTC, Fakulti Kejuruteraan, Fakulti Perniagaan dan

Perakaunan, Fakulti Sains and Tasik Varsiti. Map data @ 2018 Google.

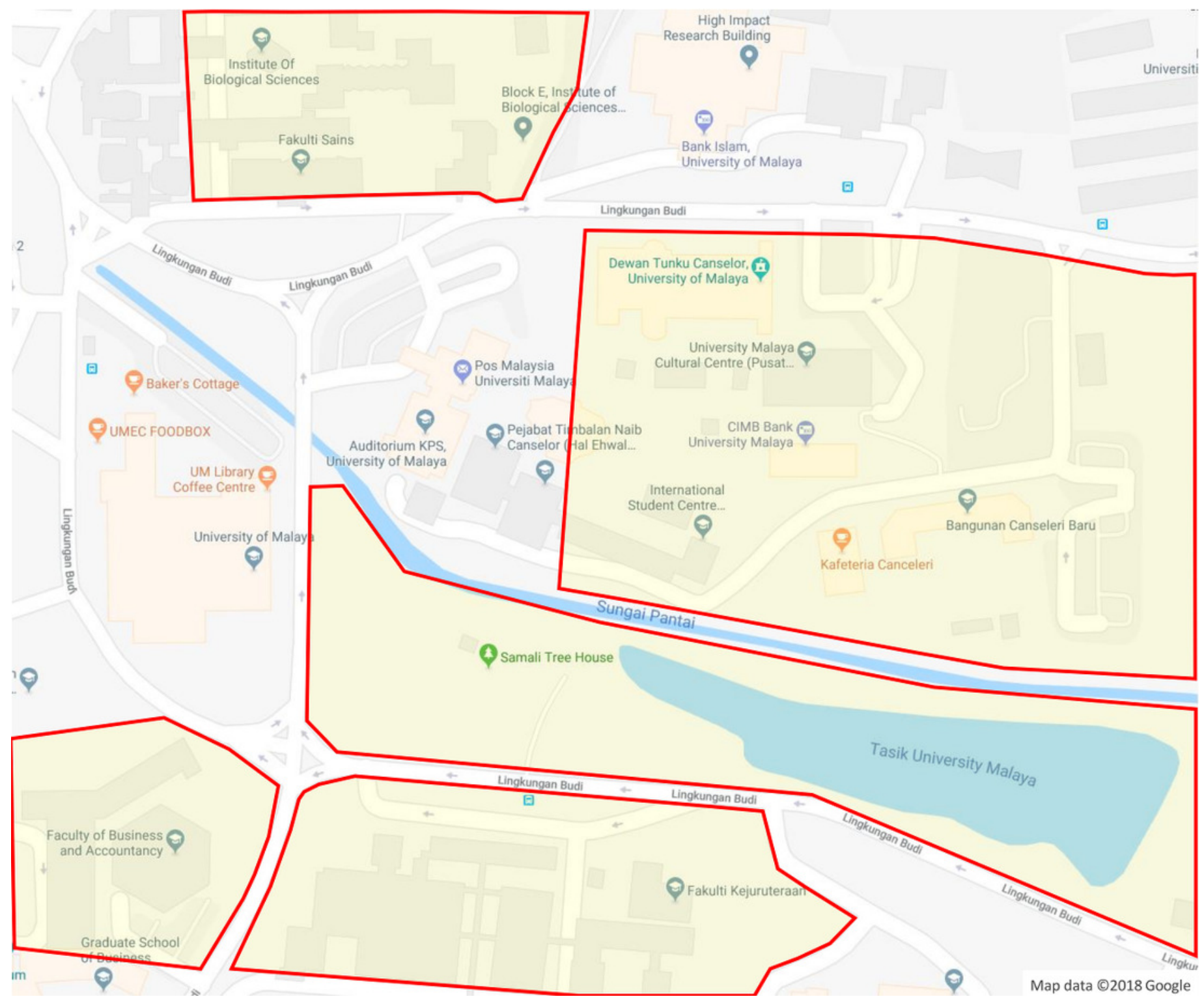


Figure 4

Proposed ontology schema.

The plant data description translated into ontology in a graph format.

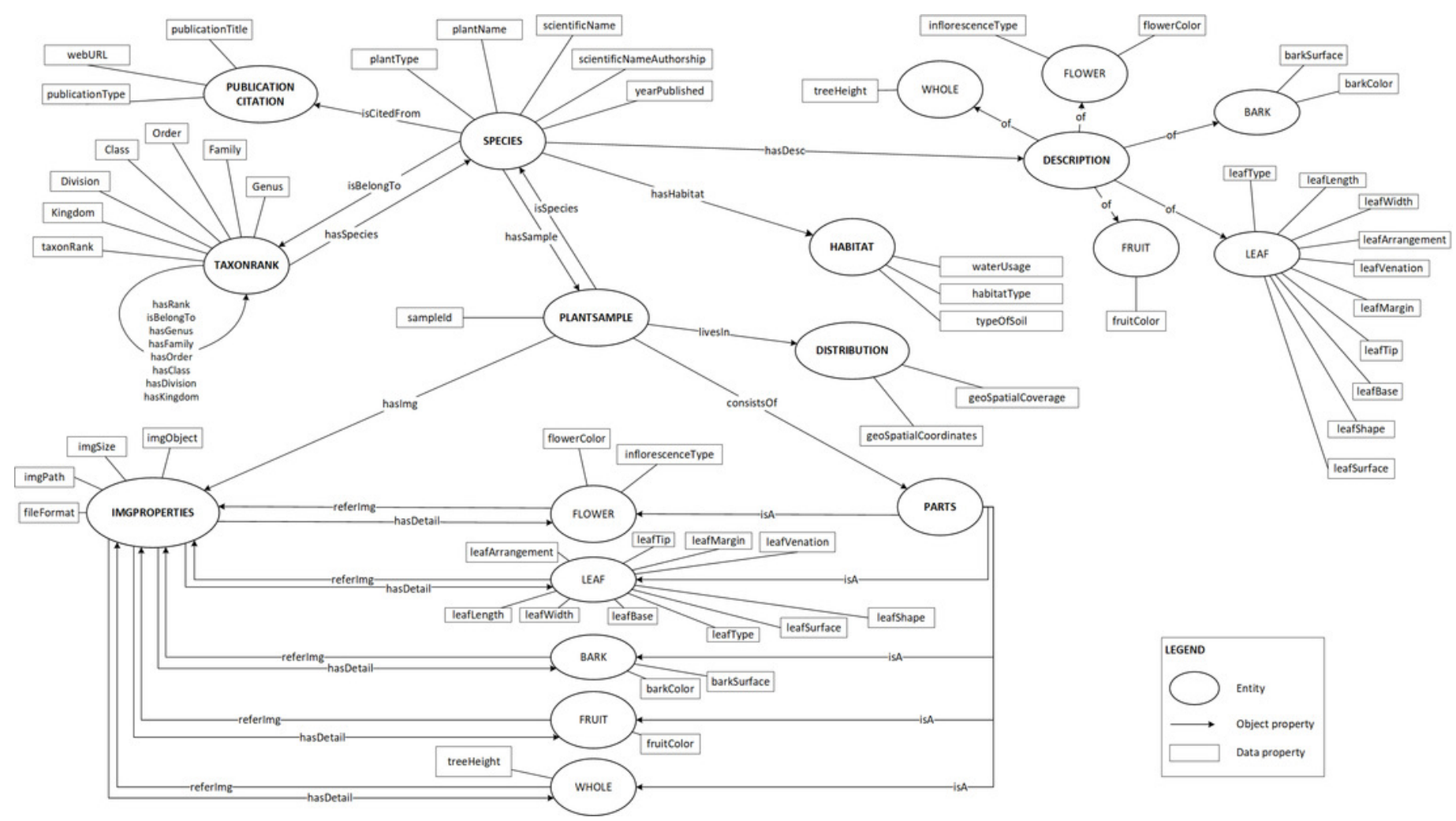


Figure 5

Top-level entities in POUM ontology.

A partial of POUM using OntoGraph plug-in in Protégé 5.2.

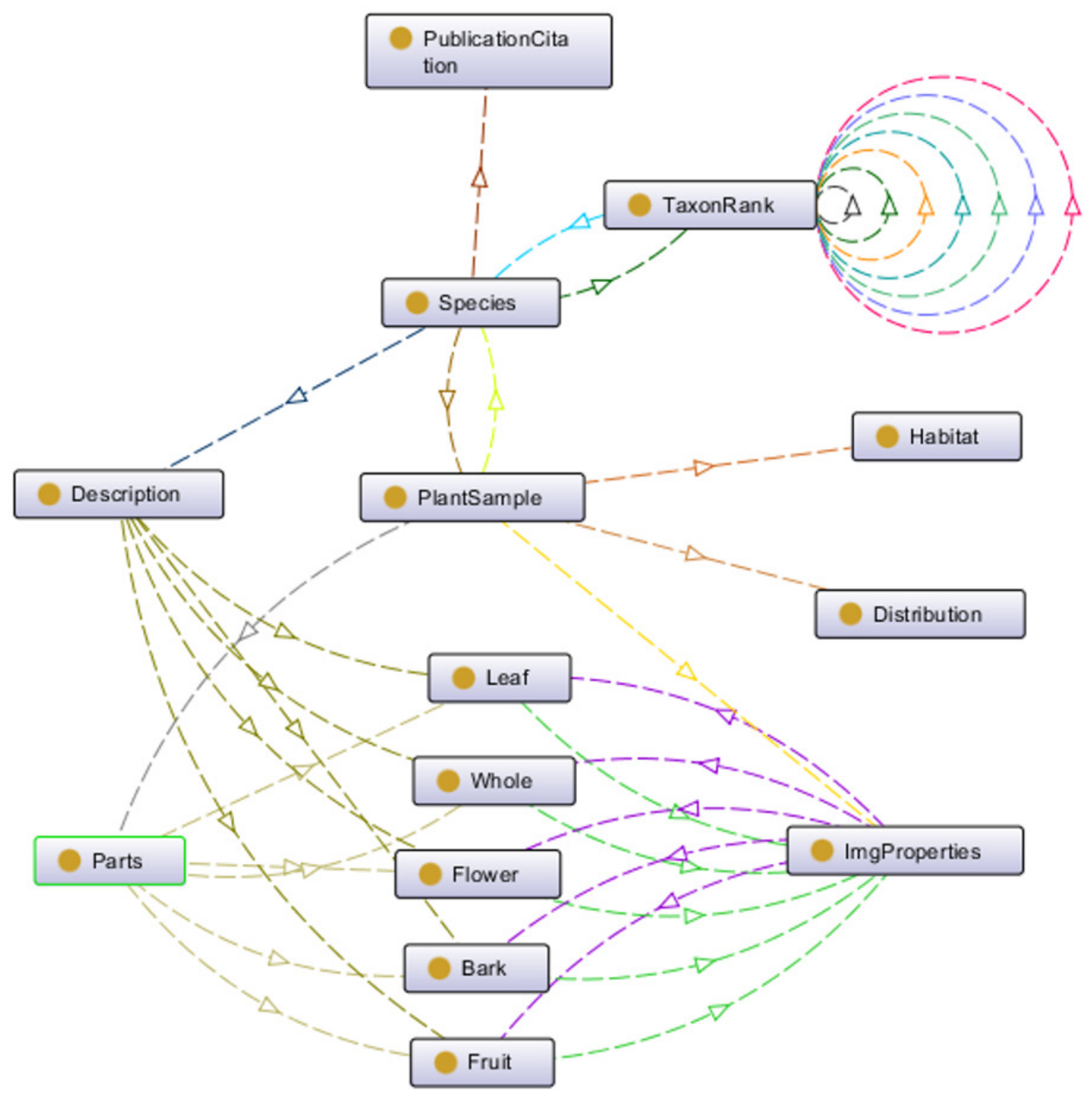




\section{Figure 6}

An example of images and description of Saraca thaipingensis sample.

(A) Digital images representing the $S$. thaipingensis sample by a whole tree and its parts, namely leaves, bark, flower and fruit. (B) Unique ID, and location data of $S$. thaipingensis sample. (C) Description of characteristics of $S$. thaipingensis sample. Photo credit: The photo archive at http://103.18.1.10:8080/plantvizl. 


\section{A}

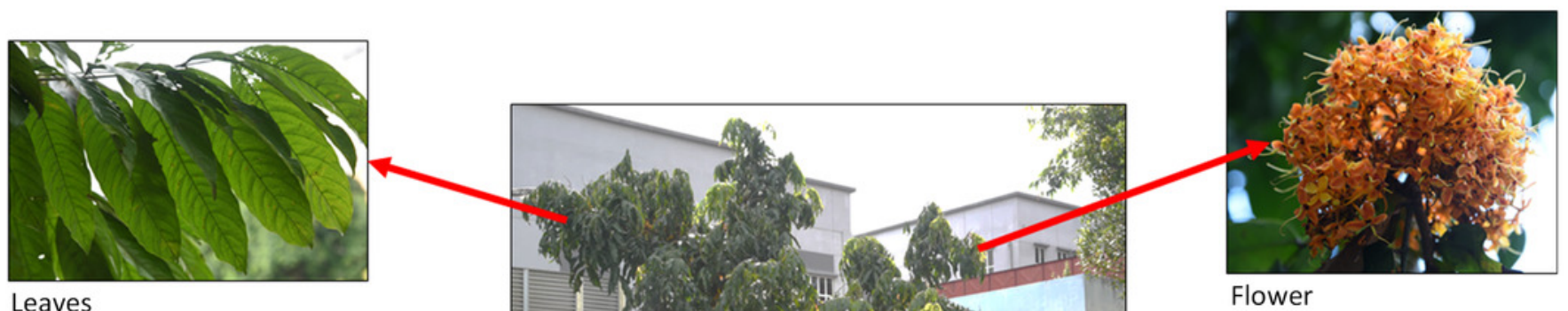

Leaves

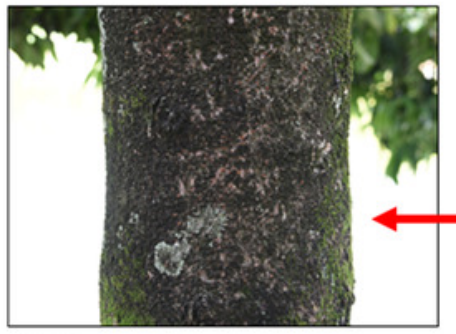

Bark

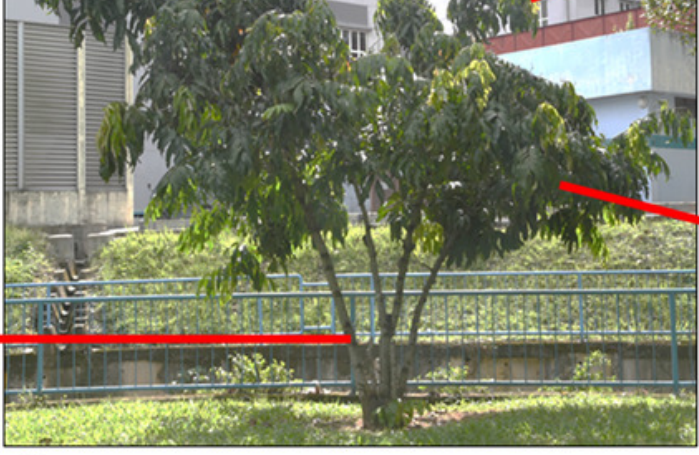

Flower

Tree

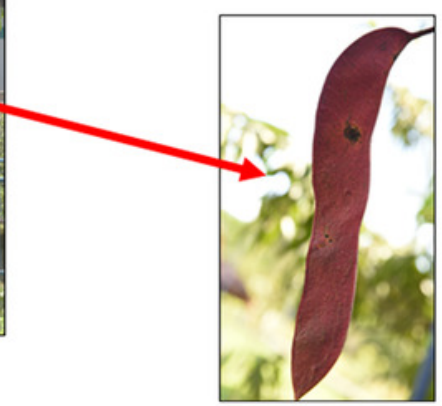

Fruit

B

\begin{tabular}{|l|l|}
\hline Sample ID & SarTha001 \\
\hline geoSpatialCoordinates & $3.1198,101.6558$ \\
\hline geoSpatialCoverage & Tasik Varsiti UM \\
\hline
\end{tabular}

C

\begin{tabular}{|l|l|l|}
\hline Plant Part & Characteristics & Data \\
\hline \multirow{5}{*}{ Leaf } & Type & Oblong \\
\cline { 2 - 3 } & Shape & Oblong \\
\cline { 2 - 3 } & Venation & Pinnate \\
\cline { 2 - 3 } & Arrangement & Alternate \\
\cline { 2 - 3 } & Margin & Entire \\
\cline { 2 - 3 } & Tip & Mucronate \\
\cline { 2 - 3 } & Base & Acute \\
\cline { 2 - 3 } & Width & 6 - 12 cm \\
\cline { 2 - 3 } & Length & $20-40 \mathrm{~cm}$ \\
\cline { 2 - 3 } & Surface & Smooth \\
\hline \multirow{5}{*}{ Fruit } & Colour & Purple \\
\hline \multirow{5}{*}{ Flower } & Colour & Orange \\
\cline { 2 - 3 } & Inflorescence type & Corymb \\
\cline { 2 - 3 } & Petal number & Petalless \\
\hline Bark & Surface & Smooth \\
\hline Tree & Height & 7 - 20 m \\
\hline
\end{tabular}


Figure 7

Workflow of network graph development.

Processes involve from passing ontological data to the creation of a network graph.

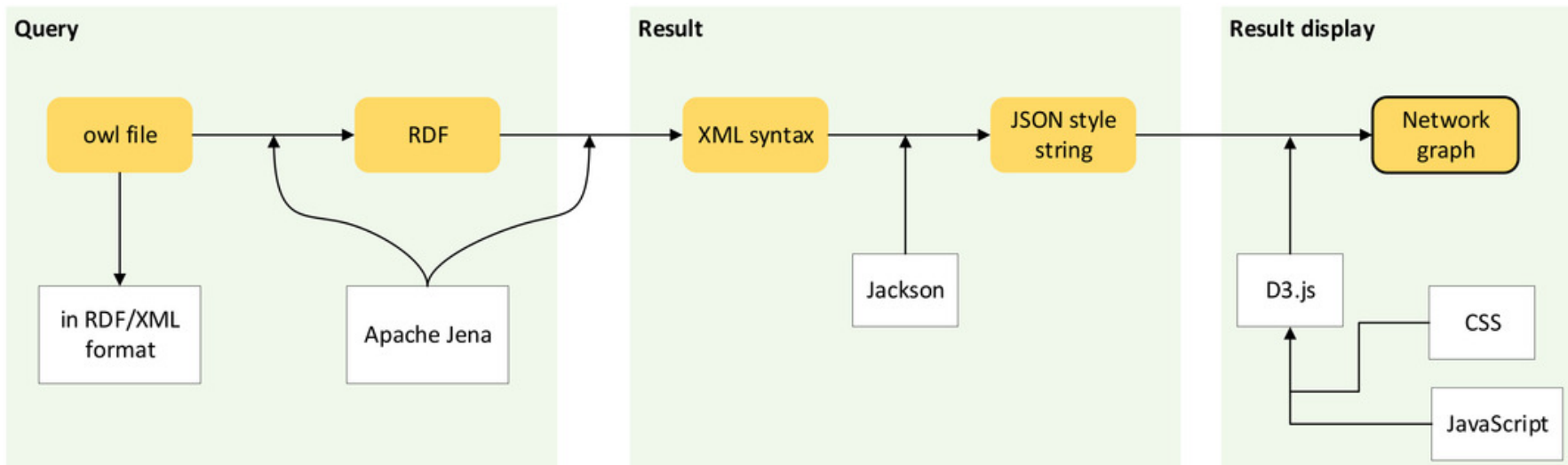


Figure 8

Sample of questionnaire for usability heuristics evaluation.

10 features were adapted from Nielson's 10 usability heuristics for $\mathrm{UI}$.

\begin{tabular}{|l|l|l|l|}
\hline & \multicolumn{1}{|c|}{ Usability Features } & Yes & No \\
\hline E1 & Status of the page is displayed (e.g. 'updates', 'news') & & \\
\hline E2 & Easy to understand the contents written in the website & & \\
\hline E3 & No irrelevant information in the website & & \\
\hline E4 & Query page is user-friendly & & \\
\hline E5 & Query page is consistent to use & & \\
\hline E6 & Guidelines are given to prevent any errors in querying & & \\
\hline E7 & Querying is easy to use even though without reading the manual page & & \\
\hline E8 & Instructions are visible when using the visualization system & & \\
\hline E9 & The error message is easy to understand & & \\
\hline E10 & 'Help' guideline on the website/visualization system is adequate & & \\
\hline
\end{tabular}


Figure 9

Sample of questionnaire for query and visualisation evaluation.

The questionnaire consists of five criteria of query evaluation and eight criteria of visualisation evaluation for the visualisation efficiency assessment.

\begin{tabular}{|c|c|c|c|c|c|c|}
\hline \multirow{2}{*}{\multicolumn{2}{|c|}{$\begin{array}{l}\text { Criteria } \\
\text { Query evaluation }\end{array}$}} & \multicolumn{5}{|c|}{ Rating score } \\
\hline & & \multirow[t]{2}{*}{1} & \multirow[t]{2}{*}{2} & \multirow[t]{2}{*}{3} & \multirow[t]{2}{*}{4} & \multirow[t]{2}{*}{5} \\
\hline Q1 & Access to a query page & & & & & \\
\hline Q2 & Perform a query & & & & & \\
\hline Q3 & Guidance on how to perform a query is adequate & & & & & \\
\hline Q4 & Number of parameter that can be queried at a time & & & & & \\
\hline Q5 & Time needed to complete a query is less than 1 minute & & & & & \\
\hline \multicolumn{2}{|c|}{ Visualization evaluation } & 1 & 2 & 3 & 4 & 5 \\
\hline V1 & Quality of visualization based on the query result & & & & & \\
\hline $\mathrm{V} 2$ & Speediness of the system to load the network graph & & & & & \\
\hline V3 & Visualization of the retrieved information is easy to understand & & & & & \\
\hline V4 & $\begin{array}{l}\text { Able to infer knowledge from the relationship of information: } \\
\bullet \text { Sample to sample } \bullet \text { Taxon to taxon } \bullet \text { Sample to taxon }\end{array}$ & & & & & \\
\hline V5 & Relevant images are shown when hovering the cursor on specific nodes & & & & & \\
\hline V6 & Information shown in visualization is sufficient & & & & & \\
\hline V7 & Access to get a complete information of plant sample & & & & & \\
\hline V8 & Error message is shown if there is no retrieved result & & & & & \\
\hline
\end{tabular}




\section{Figure 10}

\section{GUI of PlantViz.}

Main interface of PlantViz that consists of a query tool and a graphical viewer.

\section{UM Plant Knowledge}

\section{QUERY}

In PlantViz's query tool, users are able to search on any of given parameters available in the drop-down menu.

Choose any parameter, key in a search keyword or choose from the options given, and click Search to perform the query.

\begin{tabular}{l|l|}
\hline Search for : & Choose option \\
\hline Choose option \\
\hline $\begin{array}{l}\text { Scientific Name } \\
\text { Family Name } \\
\text { Location } \\
\text { Water Usage }\end{array}$ \\
\hline
\end{tabular}




\title{
Figure 11
}

\section{Ontological data in the form of a RDF graph data model.}

\author{
A fragment of the ontological data in RDF serialisation format.
}

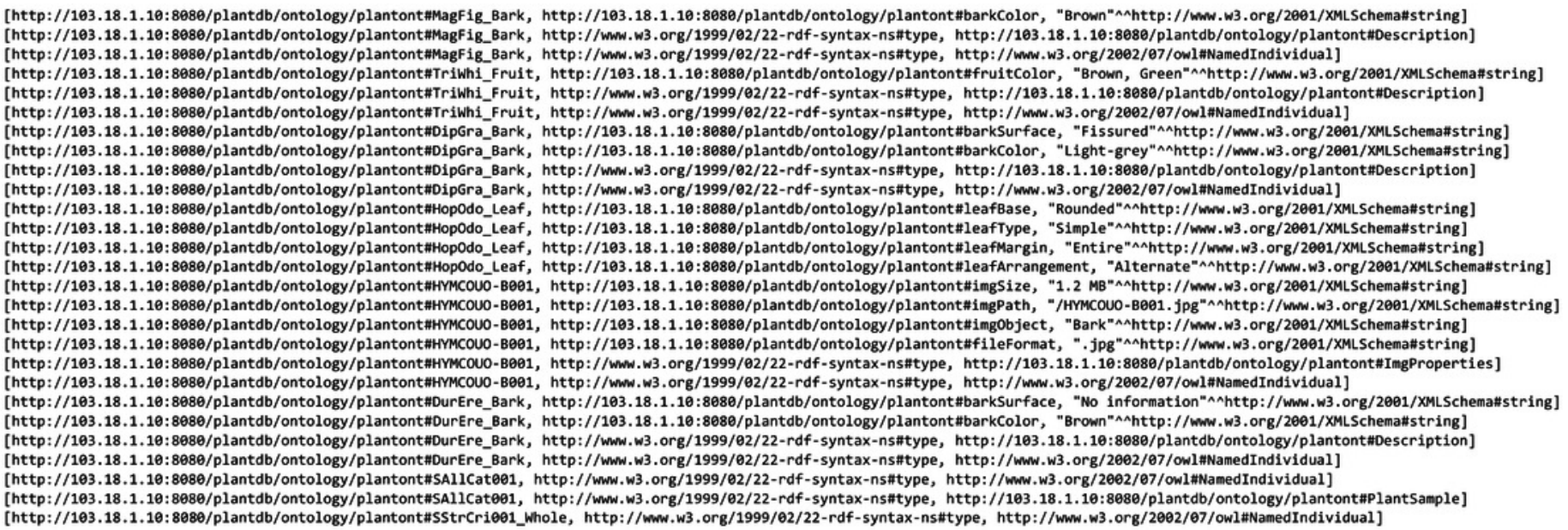


Figure 12

An example of query result in XML and JSON formats.

(A) The results generated by Jena is in XML format. (B) The results in JSON format that show the relationship between the data.

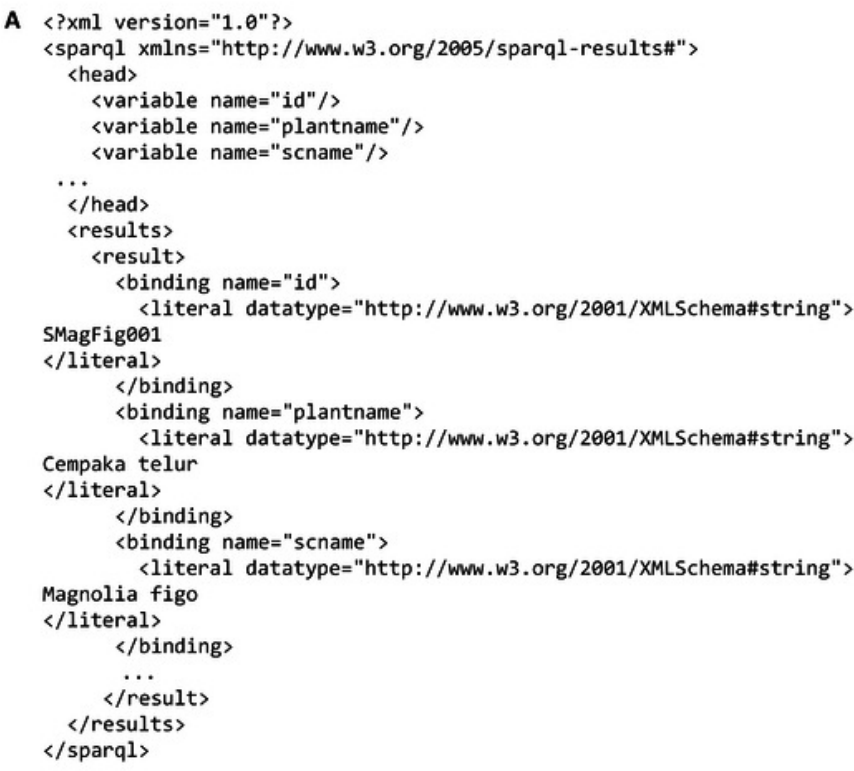

B \{ "id": "1",

"name": "Magnolia figo",

"con": "Scientific Name",

"size:": 2000

"children": [

\{

"id": "2",

"name": "General Information",

"size": 2000

"children":

f

"id": "3",

"name": "Cempaka telur",

"con": "Common Name",

\},

"size": 2000

"id": "4",

"name": "Fertile loamy, well-drained soils",

"con": "Soil",

"id": "5",

"name": "Moderate",

"con": "Water Usage",

\}

"size": 2000

\},

] 


\section{Figure 13}

PlantViz data visualisation.

An example of visualisation generated in PlantViz using the Scientific Name parameter, Magnolia figo.

\section{RESULT}

Result for Scientific Name : Magnolia figo

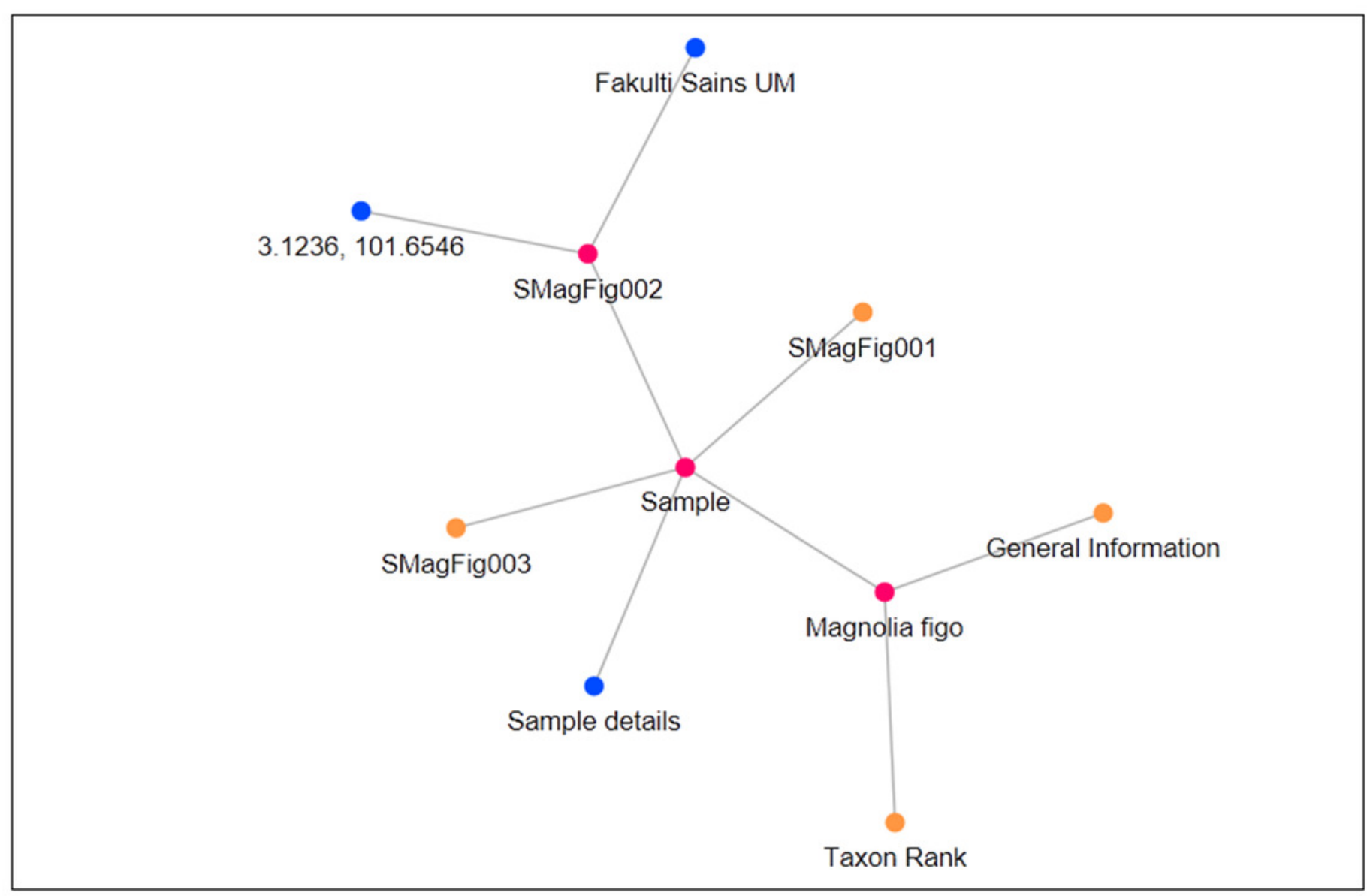




\section{Figure 14}

Examples of data visualisation for the four query parameters.

(A) Query parameter - Scientific Name. (B) Query parameter - Family Name. (C) Query parameter - Location. (D) Query parameter - Water Usage.

A RESULT

Result for Scientific Name : Hura crepitans

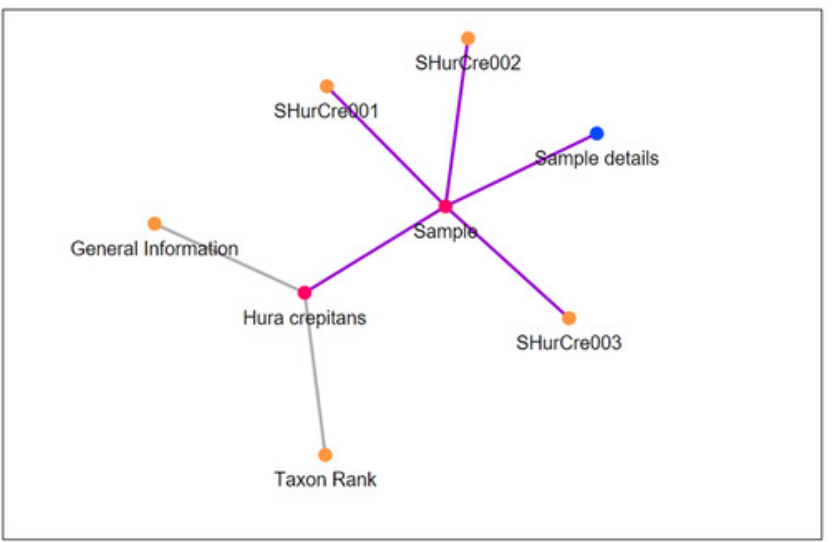

C RESULT

Result for Location : DTC UM

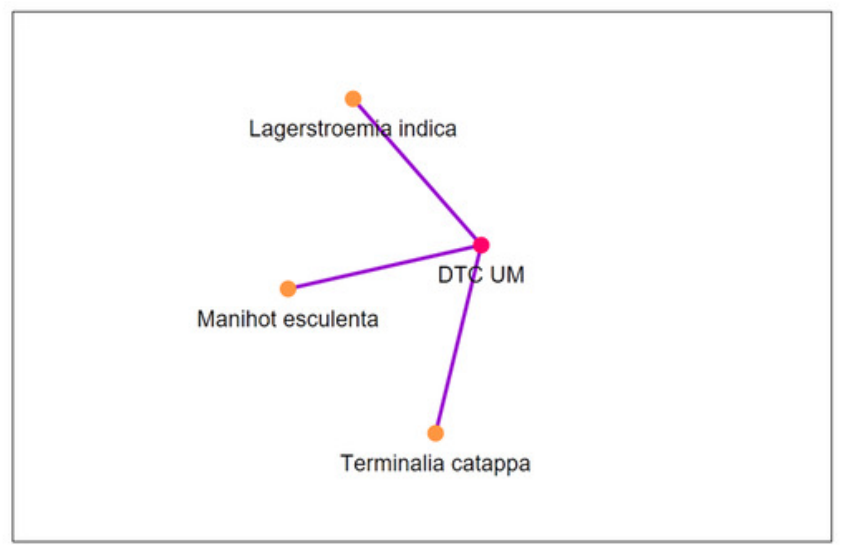

B RESULT

Result for Family Name : Acanthaceae

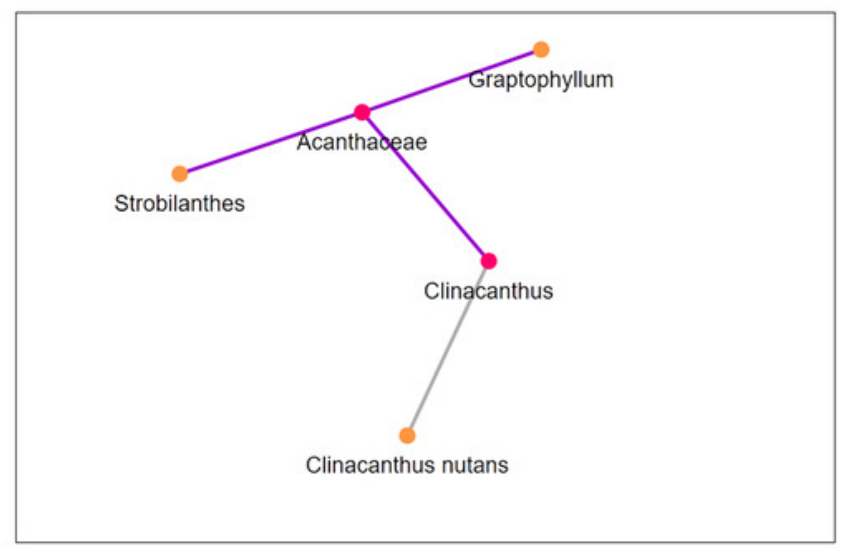

D RESULT

Result for Water Usage : Low to moderate

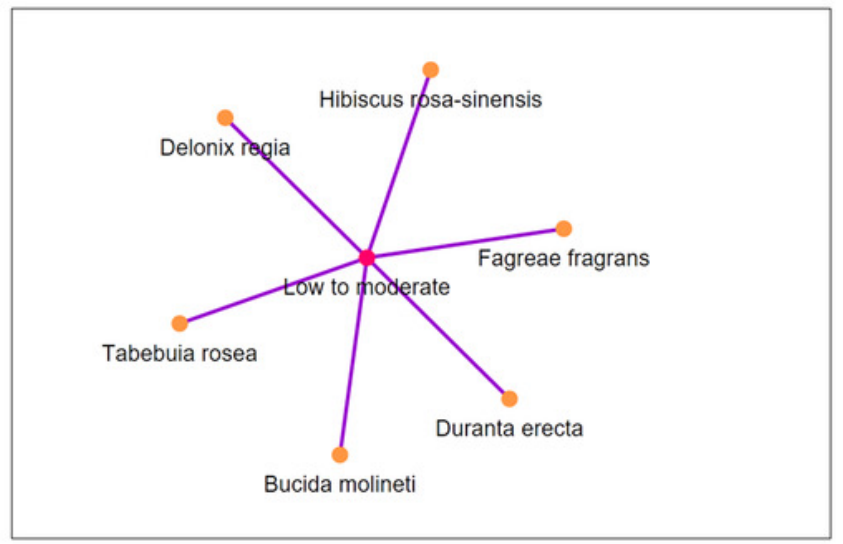




\section{Figure 15}

Interactive elements in PlantViz's graphical viewer.

(A) view node label. (B) highlight node links. (C) expand or shrink group of nodes. (D) sample information page. (E) thumbnail images of plant sample. Photo credit: The photo archive at http://103.18.1.10:8080/plantviz/. 

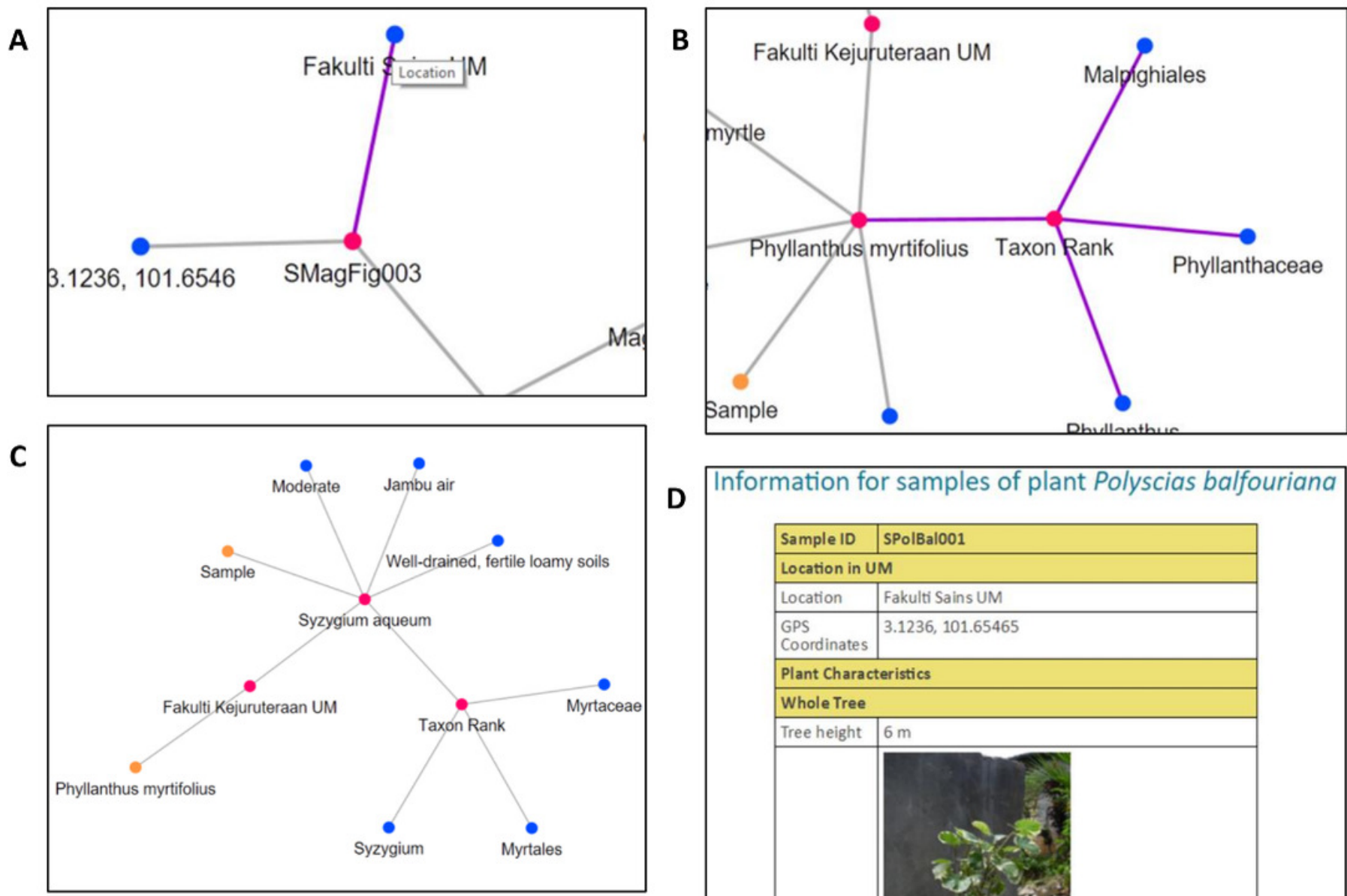

D Information for samples of plant Polyscias balfouriana

E

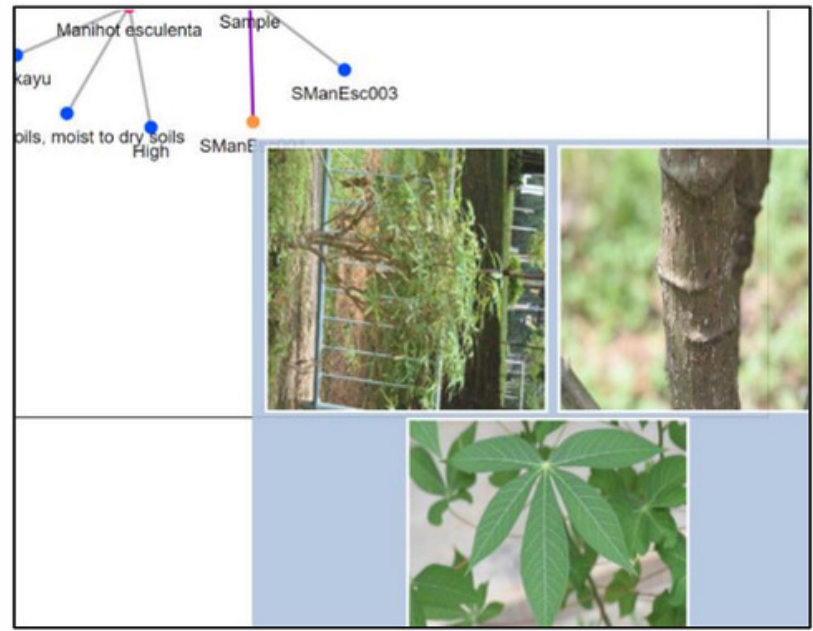

\begin{tabular}{|c|c|}
\hline Sample ID & SPolBal001 \\
\hline \multicolumn{2}{|c|}{ Location in UM } \\
\hline Location & Fakulti Sains UM \\
\hline $\begin{array}{l}\text { GPS } \\
\text { Coordinates }\end{array}$ & $3.1236,101.65465$ \\
\hline \multicolumn{2}{|c|}{ Plant Characteristics } \\
\hline \multicolumn{2}{|l|}{ Whole Tree } \\
\hline Tree height & $6 \mathrm{~m}$ \\
\hline \multirow{2}{*}{\multicolumn{2}{|c|}{$\begin{array}{l}\text { Sample } \\
\text { image } \\
\text { Leaf }\end{array}$}} \\
\hline & \\
\hline \multicolumn{2}{|r|}{ Compound } \\
\hline Length & $5-20 \mathrm{~cm}$ \\
\hline Width & $5-20 \mathrm{~cm}$ \\
\hline Arrangement & Whorled \\
\hline Venation & Pinnate \\
\hline Margin & Crenate \\
\hline Tip & Emarginate \\
\hline Base & Cordate \\
\hline Shape & Rhomboid \\
\hline Surface & null \\
\hline
\end{tabular}




\section{Figure 16}

Analysis of usability heuristics evaluation by expert and novice users.

The bar chart shows the total number of responses that rated 'Yes' and 'No' for each feature in the usability heuristics evaluation, as per the (A) expert users, and (B) novice users. 


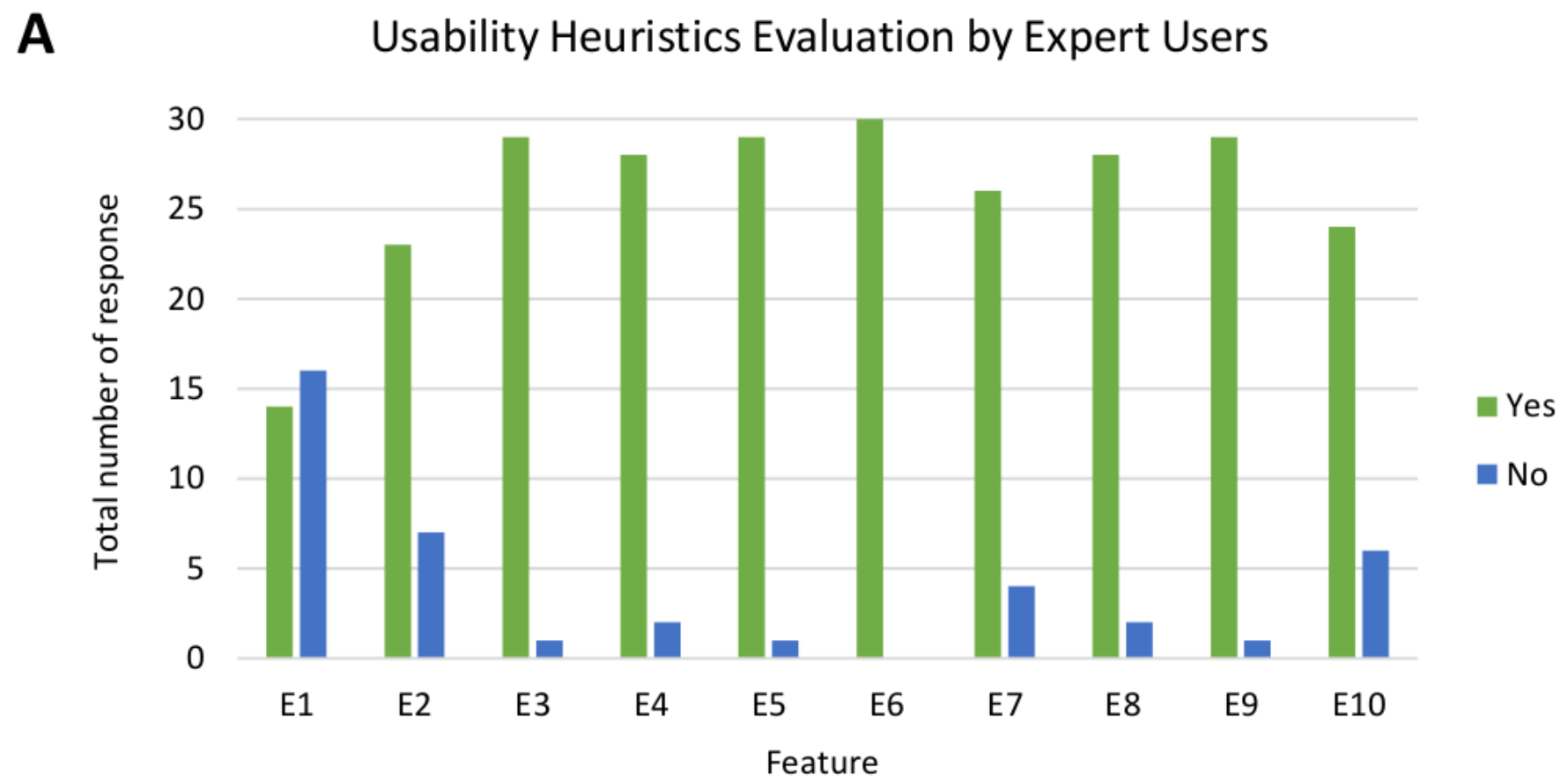

B Usability Heuristics Evaluation by Novice Users

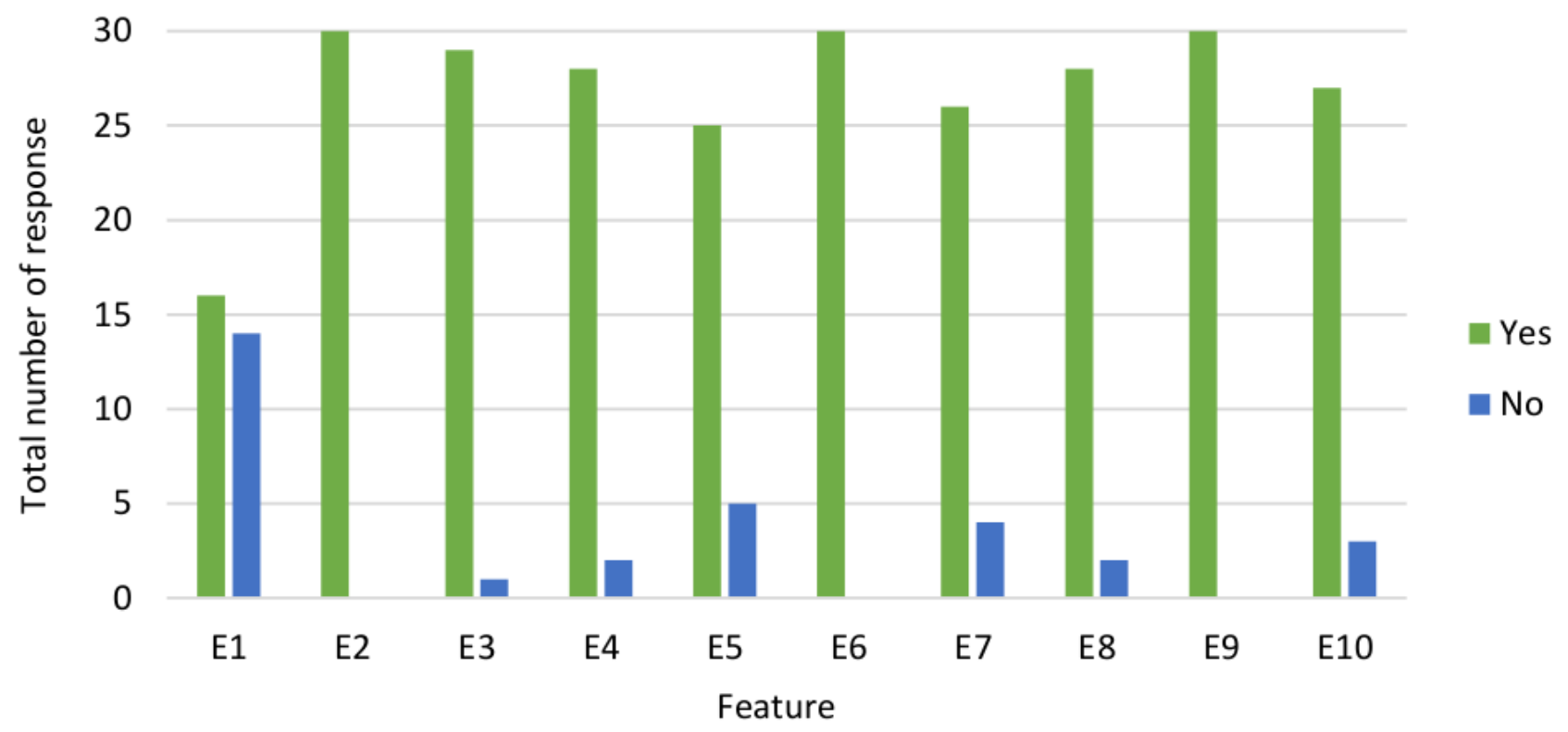




\section{Figure 17}

Analysis of query evaluation by expert and novice users.

The bar chart shows the total number of responses for each rating of all four cases by $(A)$ expert users, (B) novice users. 
A

Query Evaluation by Expert Users

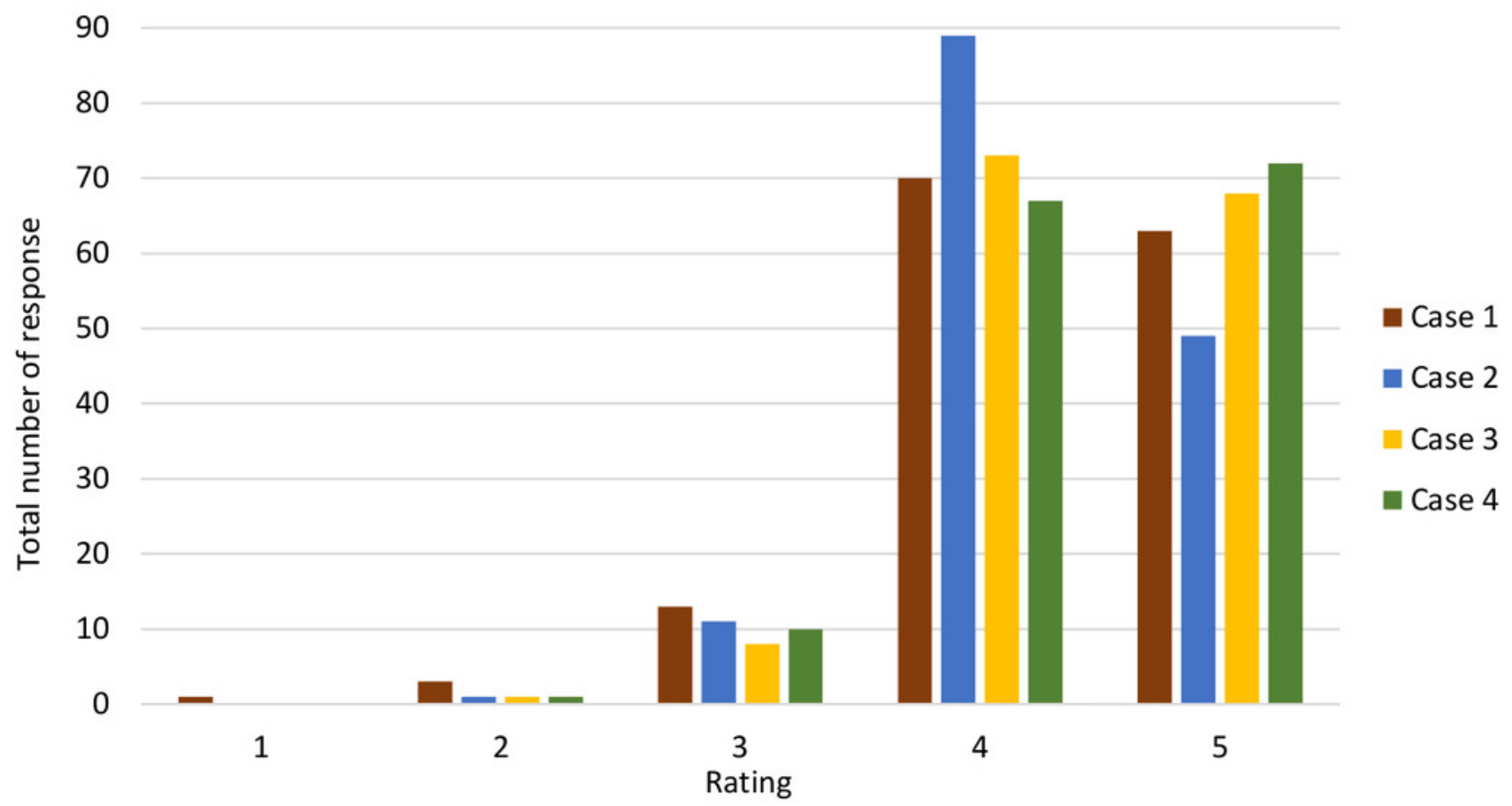

B Query Evaluation by Novice Users

90

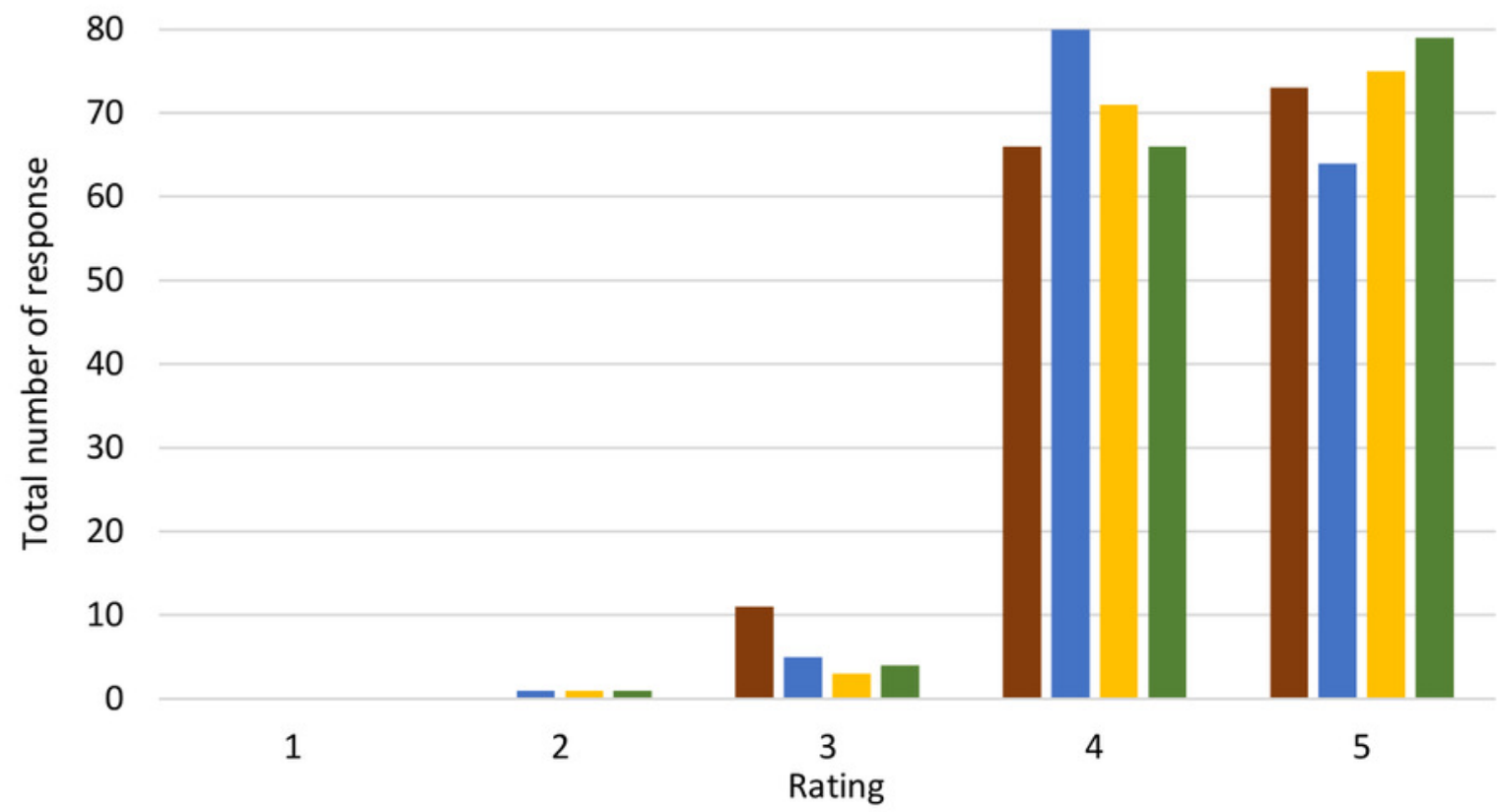

- Case 1

- Case 2

Case 3

- Case 4 


\section{Figure 18}

Analysis of visualisation evaluation by expert and novice users.

The bar chart shows the total number of responses for each rating for all four cases by (A) expert users, (B) novice users. 
A

Visualization Evaluation by Expert Users

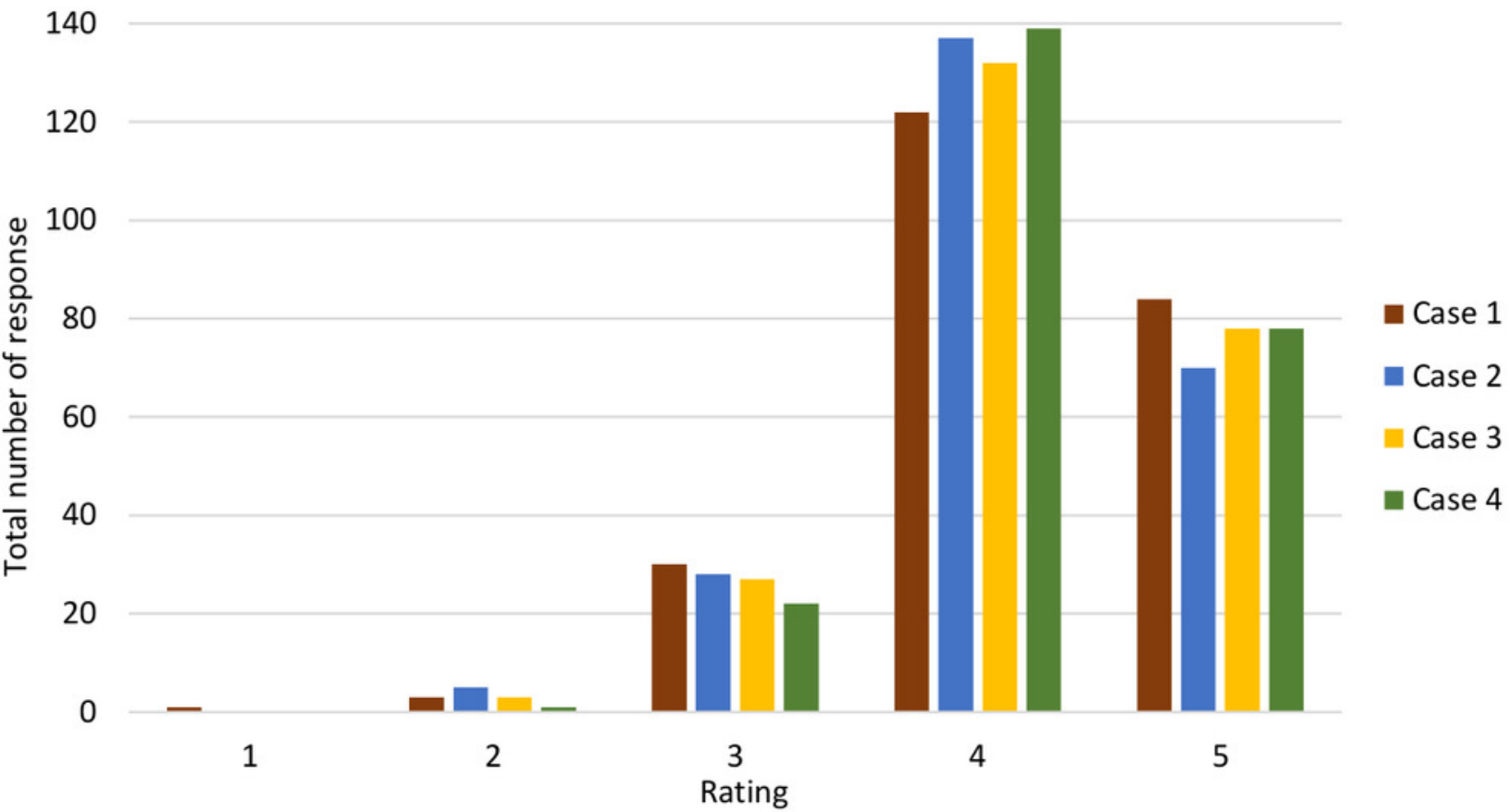

B

Visualization Evaluation by Novice Users

140

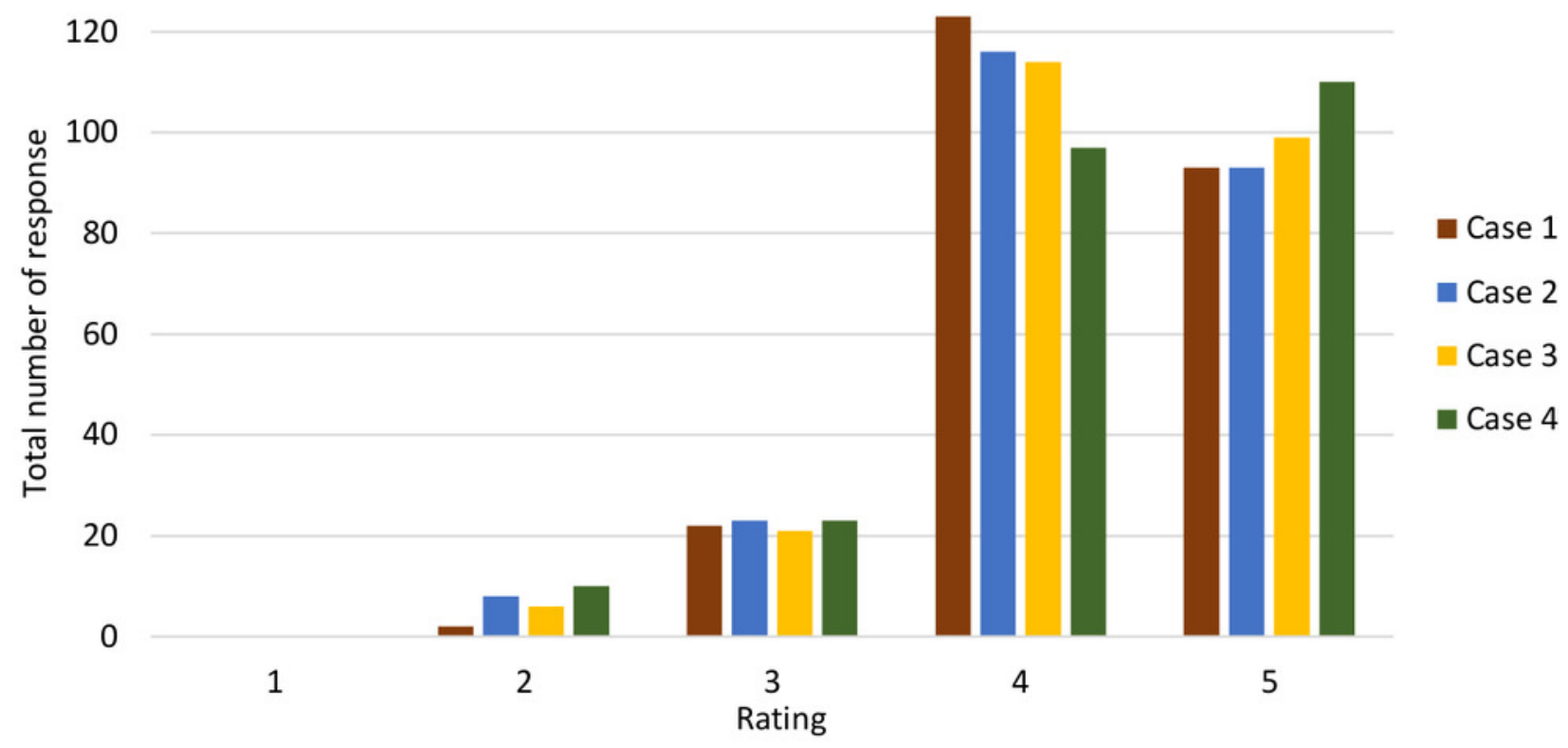

